\title{
Natural Products as Sources of New Drugs over the Last 25 Years $^{\perp}$
}

\author{
David J. Newman* and Gordon M. Cragg \\ Natural Products Branch, Developmental Therapeutics Program, Division of Cancer Treatment and Diagnosis, National Cancer \\ Institute-Frederick, P.O. Box B, Frederick, Maryland 21702
}

Received October 10, 2006

\begin{abstract}
This review is an updated and expanded version of two prior reviews that were published in this journal in 1997 and 2003. In the case of all approved agents the time frame has been extended to include the $25 \frac{1}{2}$ years from $01 / 1981$ to 06/2006 for all diseases worldwide and from 1950 (earliest so far identified) to 06/2006 for all approved antitumor drugs worldwide. We have continued to utilize our secondary subdivision of a "natural product mimic" or "NM" to join the original primary divisions. From the data presented, the utility of natural products as sources of novel structures, but not necessarily the final drug entity, is still alive and well. Thus, in the area of cancer, over the time frame from around the 1940 s to date, of the 155 small molecules, $73 \%$ are other than "S" (synthetic), with $47 \%$ actually being either natural products or directly derived therefrom. In other areas, the influence of natural product structures is quite marked, with, as expected from prior information, the antiinfective area being dependent on natural products and their structures. Although combinatorial chemistry techniques have succeeded as methods of optimizing structures and have, in fact, been used in the optimization of many recently approved agents, we are able to identify only one de novo combinatorial compound approved as a drug in this 25 plus year time frame. We wish to draw the attention of readers to the rapidly evolving recognition that a significant number of natural product drugs/leads are actually produced by microbes and/or microbial interactions with the "host from whence it was isolated", and therefore we consider that this area of natural product research should be expanded significantly.
\end{abstract}

It is over nine years since the publication of our first, ${ }^{1}$ and three years since the second, ${ }^{2}$ analysis of the sources of new and approved drugs for the treatment of human diseases, both of which indicated that natural products continued to play a highly significant role in the drug discovery and development process.

That this influence of Nature in one guise or another has continued is shown by inspection of the information given below, where with the advantage of now over 25 years of data, we have been able to refine the system, eliminating a few duplicative entries that crept into the original data sets. In particular, as behooves authors from the National Cancer Institute (NCI), in the specific case of cancer treatments, we have gone back to consult the records of the FDA and added to these, comments from investigators who have informed us over the past two years of compounds that may have been approved in other countries and that were not captured in our earlier searches. These cancer data will be presented as a stand-alone section as well as including the last 25 years of data in the overall discussion.

As we mentioned in our 2003 review, ${ }^{2}$ the development of highthroughput screens based on molecular targets had led to a demand for the generation of large libraries of compounds to satisfy the enormous capacities of these screens. As we mentioned at that time, the shift away from large combinatorial libraries has continued, with the emphasis now being on small, focused (100 to 3000) collections that contain much of the "structural aspects" of natural products. Various names have been given to this process, including "Diversity Oriented Syntheses", ${ }^{3-6}$ but we prefer to simply say "more natural product-like", in terms of their combinations of heteroatoms and significant numbers of chiral centers within a single molecule, ${ }^{7}$ or even "natural product mimics" if they happen to be direct competitive inhibitors of the natural substrate. It should also be pointed out that Lipinski's fifth rule effectively states that the first four rules do not apply to natural products or to any molecule

${ }^{\perp}$ Dedicated to the late Dr. Kenneth L. Rinehart of the University of Illinois at Urbana-Champaign for his pioneering work on bioactive natural products.

* To whom correspondence should be addressed. Tel: (301) 846-5387. Fax: (301) 846-6178. E-mail: newmand@mail.nih.gov. that is recognized by an active transport system when considering "druggable chemical entities". ${ }^{8-10}$

Although combinatorial chemistry in one or more of its manifestations has now been used as a discovery source for approximately $70 \%$ of the time covered by this review, to date, we can find only one de novo new chemical entity (NCE) reported in the public domain as resulting from this method of chemical discovery and approved for drug use anywhere. This is the antitumor compound known as sorafenib (Nexavar, 1) from Bayer, approved by the FDA in 2005. It was known during development as BAY43-9006 and is a multikinase inhibitor, targeting several serine/ threonine and receptor tyrosine kinases (RAF kinase, VEGFR-2, VEGFR-3, PDGFR-beta, KIT, and FLT-3) and is in multiple clinical trials as both combination and single-agent therapies at the present time, a common practice once approved for one class of cancer treatment.

As mentioned by the authors in prior reviews on this topic and others, the developmental capability of combinatorial chemistry as a means for structural optimization once an active skeleton has been identified is without par. The expected surge in productivity, however, has not materialized; thus, the number of new active substances (NASs), also known as New Chemical Entities (NCEs), which we consider to encompass all molecules, including biologics and vaccines, from our data set hit a 24-year low of 25 in 2004 (though $28 \%$ of these were assigned to the ND category), with a rebound to 54 in 2005, with $24 \%$ being N or ND and $37 \%$ being biologics (B) or vaccines (V). Fortunately, however, research being conducted by groups such as Danishefsky's, Ganesan's, Nicolaou's, Porco's, Quinn's, Schreiber's, Shair's, Waldmann's, and Wipf's is continuing the modification of active natural product skeletons as leads to novel agents, so in due course, the numbers of materials developed by linking Mother Nature to combinatorial synthetic techniques should increase. This aspect, plus the potential contributions from the utilization of genetic analyses of microbes, will be discussed at the end of this review.

Against this backdrop, we now present an updated analysis of the role of natural products in the drug discovery and development process, dating from 01/1981 through 06/2006. As in our earlier 
analyses, ${ }^{1,2}$ we have consulted the Annual Reports of Medicinal Chemistry, in this case from 1984 to $2005,{ }^{11-32}$ and have produced a more comprehensive coverage of the 1981-2006 time frame through addition of data from the publication Drug News and Perspective $e^{33-49}$ and searches of the Prous Integrity database, as well as by including information from individual investigators. We also updated the biologicals section of the data set using information culled from disparate sources that culminated in a recent review (2005) on biopharmaceutical drugs. ${ }^{50}$

We have also included relevant references in a condensed form in Tables $1-5,8$, and 9; otherwise the numbers of references cited in the review would become overwhelming. In these cases, "ARMC \#\#" refers to the volume of Annual Reports in Medicinal Chemistry together with the page on which the structure(s) can be found. Similarly, "DNP \#\#" refers to the volume of Drug News and Perspective and the corresponding page(s), and an "I \#\#\#\#\#" is the accession number in the Prous Integrity database. Finally, we have used "Boyd" to refer to a review article ${ }^{51}$ on clinical antitumor agents and "M'dale" to refer to Martindale ${ }^{52}$ with the relevant page noted.

It should be noted that the "Year" header in all tables is the "Year of Introduction" of the drug. In some cases there are discrepancies between sources as to the actual year due to differences in definitions. We have generally taken the earliest year in the absence of further information.

\section{Results}

As before, we have covered only New Chemical Entities (NCEs) in the present analysis. If one reads the FDA and PhRMA Web sites, the numbers of NDA approvals are in the high tens to low hundred numbers for the last few years. If, however, one removes combinations of older drugs and old drugs with new indications, and/or improved delivery systems, then the number of true NCEs is only in the 20 s to just over 50 per year for the last five or so years (see Figures 2 and 5).

As in our earlier analyses, ${ }^{1,2}$ the data have been analyzed in terms of numbers and classified according to their origin using both the previous major categories and their subdivisions.

Major Categories of Sources. The major categories used are as follows:

"B" Biological; usually a large ( $>45$ residues) peptide or protein either isolated from an organism/cell line or produced by biotechnological means in a surrogate host.

"N" Natural product.

"ND" Derived from a natural product and is usually a semisynthetic modification.

"S" Totally synthetic drug, often found by random screening/ modification of an existing agent.

"S*" Made by total synthesis, but the pharmacophore is/was from a natural product.

"V" Vaccine.

Subcategory. "NM" Natural product mimic (see rationale and examples below).

(For amplification as to the rationales used for categorizing using the above subdivisions, the reader should consult the earlier reviews. ${ }^{1,2}$ )

In the field of anticancer therapy, the advent in 2001 of Gleevec, a protein tyrosine kinase inhibitor, was justly heralded as a breakthrough in the treatment of leukemia. This compound was classified as an "NM" on the basis of its competitive displacement of the natural substrate ATP, whose intracellular concentrations can approach $5 \mathrm{mM}$. We have continued to classify PTK and other kinase inhibitors that are approved as drugs under the "/NM" category for exactly the same reasons as elaborated in the 2003 review $^{2}$ and have continued to extend it to cover other direct inhibitors/antagonists of the natural substrate/receptor interac- tion whether obtained by direct experiment or by in silico studies followed by direct assay in the relevant system. Similarly, a number of new peptidic drug entities, though formally synthetic in nature, are simply produced by synthetic methods rather than by the use of fermentation or extraction. In some cases, an end group might have been changed for ease of recovery. In addition, a number of compounds produced totally by synthesis are, in fact, isosteres of the peptidic substrate and are thus "natural product mimics" in the truest sense of the term. For further information on this area, interested readers should consult the excellent review by Hruby. ${ }^{53}$
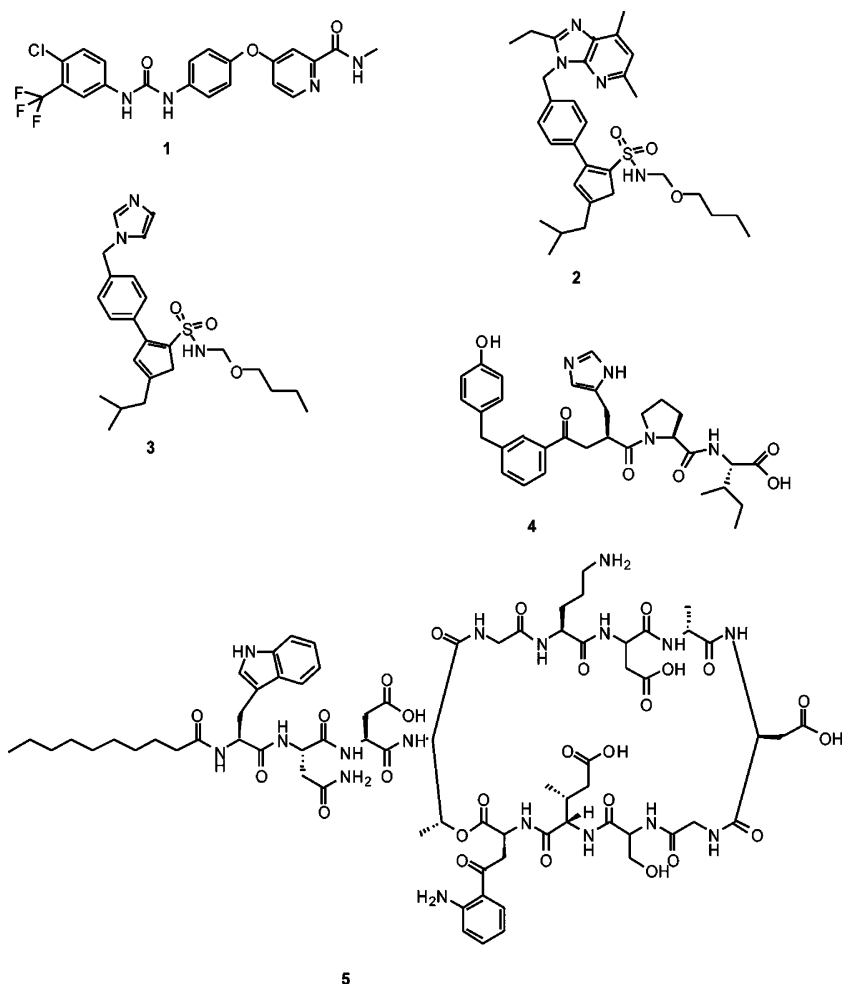

As an example of what can be found by studies around relatively simple peptidomimics of the angiotensin II structure, the recent paper of Wan et al. ${ }^{54}$ demonstrating the modification of the known but nonselective $\mathrm{AT}_{1} / \mathrm{AT}_{2}$ agonist $\mathrm{L}-162313$ (2, itself related to the sartans) into the highly selective $\mathrm{AT}_{2}$ agonist (3) (a peptidomimetic structure) led to the very recent identification of short pseudopeptides exemplified by $\mathbf{4}$, which is equipotent (binding affinity $=$ $500 \mathrm{pM}$ ) with angiotensin II and has a better than 20000 -fold selectivity versus $\mathrm{AT}_{1}$, whereas angiotensin II has only a 5-fold binding selectivity in the same assay. ${ }^{55}$ It will be interesting to see if any compounds such as these will end up as cardiovascular agents since it has been demonstrated that activation of the $\mathrm{AT}_{2}$ receptor affects cardiac remodeling and leads to reduced blood pressure. ${ }^{56}$

In the area of modifications of natural products by combinatorial methods to produce entirely different compounds that may bear little if any resemblance to the original, but are legitimately assignable to the "NM" category, citations are given in previous reviews. ${ }^{3,57-64}$ In addition, one should consult the recent reports from Waldmann's group ${ }^{65,66}$ and those by Ganesan, ${ }^{67}$ Shang and Tan, ${ }^{68}$ Constantino, ${ }^{69}$ and Violette et al. ${ }^{70}$ on the use of privileged structures as skeletons around which to build libraries. Another paper of interest in this regard is the editorial by Macarron from GSK, ${ }^{9}$ as this may be the first time where data from industry on the results of HTS screens of combichem libraries versus potential targets were reported with a discussion of lead discovery rates. In this paper, Macarron reemphasizes the fifth Lipinski rule, which is often ignored; "natural products do not obey the other four". 
Table 1. New Chemical Entities and Medical Indications by Source of Compound 01/1981-06/2006 ${ }^{a, b}$

\begin{tabular}{|c|c|c|c|c|c|c|c|c|c|}
\hline \multirow[b]{2}{*}{ indication } & \multirow[b]{2}{*}{ total } & \multicolumn{8}{|c|}{ origin of drug } \\
\hline & & B & $\mathrm{N}$ & ND & $\mathrm{S}$ & $\mathrm{S} / \mathrm{NM}$ & $S^{*}$ & $\mathrm{~S} * / \mathrm{NM}$ & $\mathrm{V}$ \\
\hline analgesic & 16 & & 1 & & 11 & 2 & 2 & & \\
\hline anesthetic & 5 & & & & 5 & & & & \\
\hline anti-Alzheimer's & 4 & & 1 & & & 3 & & & \\
\hline anti-Parkinsonism & 12 & & & 2 & 1 & 5 & & 4 & \\
\hline antiallergic & 16 & & 1 & 3 & 12 & & & & \\
\hline antianginal & 5 & & & & 5 & & & & \\
\hline antiarrhythmic & 16 & & 1 & & 13 & & & 2 & \\
\hline antiarthritic & 15 & 5 & & 1 & 3 & 6 & & & \\
\hline antiasthmatic & 14 & 1 & & 3 & 2 & 6 & & 2 & \\
\hline antibacterial & 109 & & 10 & 64 & 23 & & & 1 & 11 \\
\hline anticancer & 100 & 17 & 9 & 25 & 18 & 12 & 11 & 6 & 2 \\
\hline anticoagulant & 17 & 4 & & 12 & & & 1 & 0 & \\
\hline antidepressant & 22 & & & & 7 & 13 & & 2 & \\
\hline antidiabetic & 32 & 18 & 1 & 4 & 4 & 4 & 1 & & \\
\hline antiemetic & 10 & & & & 1 & 1 & & 8 & \\
\hline antiepileptic & 11 & & & 2 & 6 & & 2 & 1 & \\
\hline antifungal & 29 & 1 & & 3 & 22 & 3 & & & \\
\hline antiglaucoma & 13 & & & 4 & & 5 & 1 & 3 & \\
\hline antihistamine & 12 & & & & 12 & & & & \\
\hline antihyperprolactinemia & 4 & & & 4 & & & & & \\
\hline antihypertensive & 77 & & & 2 & 27 & 14 & 2 & 32 & \\
\hline antiinflammatory & 51 & 1 & & 13 & 37 & & & & \\
\hline antimigraine & 10 & & & & 2 & 1 & & 7 & \\
\hline antiobesity & 4 & & & 1 & & 3 & & & \\
\hline antiparasitic & 14 & & 2 & 5 & 4 & & 2 & & 1 \\
\hline antipsoriatic & 7 & 2 & & 3 & & & 1 & 1 & \\
\hline antipsychotic & 7 & & & & 3 & 2 & & 2 & \\
\hline antithrombotic & 28 & 13 & 1 & 5 & 2 & 5 & & 2 & \\
\hline antiulcer & 32 & 1 & 1 & 12 & 18 & & & & \\
\hline antiviral & 78 & 12 & & 2 & 7 & 1 & 20 & 11 & 25 \\
\hline anxiolytic & 10 & & & & 8 & 2 & & & \\
\hline benign prostatic hypertrophy & 4 & & 1 & 1 & 1 & 1 & & & \\
\hline bronchodilator & 8 & & & 2 & & & & 6 & \\
\hline calcium metabolism & 17 & & & 8 & 8 & 1 & & & \\
\hline cardiotonic & 13 & & & 3 & 2 & 3 & & 5 & \\
\hline chelator \& antidote & 5 & & & & 4 & 1 & & & \\
\hline contraception & 7 & & & 7 & & & & & \\
\hline diuretic & 5 & & & & 4 & 1 & & & \\
\hline gastroprokinetic & 4 & & & & 1 & 2 & & 1 & \\
\hline hematopoiesis & 6 & 6 & & & & & & & \\
\hline hemophilia & 11 & 11 & & & & & & & \\
\hline hormone & 22 & 12 & & 10 & & & & & \\
\hline hormone replacement therapy & 8 & & & 8 & & & & & \\
\hline hypnotic & 12 & & & & 12 & & & & \\
\hline hypocholesterolemic & 11 & & 3 & 1 & 2 & & & 5 & \\
\hline hypolipidemic & 8 & & 1 & & 7 & & & & \\
\hline immunomodulator & 4 & 2 & 1 & 1 & & & & & \\
\hline immunostimulant & 10 & 4 & 3 & 2 & 1 & & & & \\
\hline immunosuppressant & 12 & 4 & 5 & 3 & & & & & \\
\hline male sexual dysfunction & 4 & & & & & & & 4 & \\
\hline multiple sclerosis & 4 & 3 & & & & & 1 & & \\
\hline muscle relaxant & 10 & & & 4 & 2 & 1 & 3 & & \\
\hline neuroleptic & 9 & & & & 1 & 6 & & 2 & \\
\hline nootropic & 8 & & & 3 & 5 & & & & \\
\hline osteoporosis & 4 & 2 & & 1 & 1 & & & & \\
\hline platelet aggregation inhibitor & 4 & & & 3 & & 1 & & & \\
\hline respiratory distress syndrome & 6 & 3 & 1 & & 1 & 1 & & & \\
\hline urinary incontinence & 4 & & & & 2 & 2 & & & \\
\hline vasodilator & 5 & & & 3 & 2 & & & & \\
\hline vulnerary & 5 & 2 & & 2 & 1 & & & & \\
\hline grand total & 1010 & 124 & 43 & 232 & 310 & 108 & 47 & 107 & 39 \\
\hline
\end{tabular}

${ }^{a}$ Where there were $\leq 3$ NCEs per indication in the time frame 01/1981-06/2006, the number of NCEs totaled 174. These were assignable as $\mathrm{B}, 41 ; \mathrm{N}, 12 ; \mathrm{ND}, 38 ; \mathrm{S}, 54 ; \mathrm{S} / \mathrm{NM}, 10 ; \mathrm{S}^{*}, 5 ; \mathrm{S}^{*} / \mathrm{NM}, 7 ; \mathrm{V}, 7 .{ }^{b}$ The indications for these 174 drugs are as follows: 5 alpha-reductase inhibitor, ADHD, CNS stimulant, COPD, Crohn's disease, Fabry's disease, Gaucher's disease, IBS, Lyme disease, MI, acute, MMRC, PCP/toxoplasmosis, Pompe's disease, abortifacient, acromelagy, actinic keratoses, adjuvant/colorectal cancer, alcohol deterrent, anabolic metabolism, analeptic, anemia, angina, anti-sickle cell anemia, antiacne, antiathersclerotic, anticholelithogenic, anticonvulsant, antidiarrheal, antidote, antiemphysemic, antiestrogenic, antihyperuricemia, antihypotensive, antinarcolepsy, antinarcotic, antinauseant, antiperistaltic, antiprogestogenic, antirheumatic, antisecretory, antisepsis, antiseptic, antispasmodic, antispastic, antitussive, antityrosinaemia, antixerostomia, benzodiazepine antagonist, beta-lactamase inhibitor, blepharospasm, bone disorders, bone morphogenesis, bowel evacuant, cardioprotective, cardiovascular disease, cervical dystonia, chelator, choleretic, chronic idiopathic constipation, cognition enhancer, congestive heart failure, cystic fibrosis, cytoprotective, diabetic foot ulcers, digoxin toxicity, diphtheria-pertussistetanus, dysuria, enzyme, erythropoiesis, expectorant, gastroprotectant, genital warts, hematological, hemostatic, hepatoprotectant, hyperammonemia, homocystinuria, hyperparathyroidism, hyperphenylalaninemia, hyperphosphatemia, hypoammonuric, hypocalciuric, hypogonadism, iron chelator, joint lubricant, lipoprotein disorders, macular degeneration, mucolytic, mucopolysaccharidosis, mucositis, myleodysplasia, narcolepsy, nasal decongestant, neuropathic pain, neuroprotective, opiate detoxification, osteoarthritis, ovulation, pancreatic disorders, pancreatitis, pertussis, photosensitizer, pituitary disorders, porphyria, premature birth, progestogen, psychostimulant, purpura fulminans, rattlesnake antivenom, reproduction, restenosis, sclerosant, secondary hyperthryoidism, sedative, skin photodamage, smoking cessation, strabismus, subarachnoid hemorrhage, thrombocytopenia, treatment of GH deficiency, ulcerative colitis, urea cycle disorders, urolithiasis. 
Table 2. Antibacterial Drugs from 01/1981 to 06/2006 Organized Alphabetically by Generic Name within Source

\begin{tabular}{|c|c|c|c|c|c|}
\hline generic name & trade name & year introduced & reference & page & source \\
\hline RV-11 & Zalig & 1989 & ARMC 25 & 318 & $\mathrm{~N}$ \\
\hline carumonam & Amasulin & 1988 & ARMC 24 & 298 & $\mathrm{~N}$ \\
\hline daptomycin & Cubicin & 2003 & ARMC 39 & 347 & $\mathrm{~N}$ \\
\hline fosfomycin trometamol & Monuril & 1988 & I 112334 & & $\mathrm{~N}$ \\
\hline isepamicin & Isepacin & 1988 & ARMC 24 & 305 & $\mathrm{~N}$ \\
\hline micronomicin sulfate & Sagamicin & 1982 & I 091082 & & $\mathrm{~N}$ \\
\hline miokamycin & Miocamycin & 1985 & ARMC 21 & 329 & $\mathrm{~N}$ \\
\hline mupirocin & Bactroban & 1985 & ARMC 21 & 330 & $\mathrm{~N}$ \\
\hline netilimicin sulfate & Netromicine & 1981 & I 070366 & & $\mathrm{~N}$ \\
\hline teicoplanin & Targocid & 1988 & ARMC 24 & 311 & $\mathrm{~N}$ \\
\hline apalcillin sodium & Lumota & 1982 & I 091130 & & ND \\
\hline arbekacin & Habekacin & 1990 & ARMC 26 & 298 & ND \\
\hline aspoxicillin & Doyle & 1987 & ARMC 23 & 328 & ND \\
\hline astromycin sulfate & Fortimicin & 1985 & ARMC 21 & 324 & ND \\
\hline azithromycin & Sunamed & 1988 & ARMC 24 & 298 & ND \\
\hline aztreonam & Azactam & 1984 & ARMC 20 & 315 & ND \\
\hline biapenem & Omegacin & 2002 & ARMC 38 & 351 & ND \\
\hline cefbuperazone sodium & Tomiporan & 1985 & ARMC 21 & 325 & ND \\
\hline cefcapene pivoxil & Flomox & 1997 & ARMC 33 & 330 & ND \\
\hline cefdinir & Cefzon & 1991 & ARMC 27 & 323 & ND \\
\hline cefditoren pivoxil & Meiact & 1994 & ARMC 30 & 297 & ND \\
\hline cefepime & Maxipime & 1993 & ARMC 29 & 334 & ND \\
\hline cefetamet pivoxil $\mathrm{HCl}$ & Globocef & 1992 & ARMC 28 & 327 & ND \\
\hline cefixime & Cefspan & 1987 & ARMC 23 & 329 & ND \\
\hline cefmenoxime $\mathrm{HCl}$ & Tacef & 1983 & ARMC 19 & 316 & ND \\
\hline cefminox sodium & Meicelin & 1987 & ARMC 23 & 330 & ND \\
\hline cefodizime sodium & Neucef & 1990 & ARMC 26 & 300 & ND \\
\hline cefonicid sodium & Monocid & 1984 & ARMC 20 & 316 & ND \\
\hline cefoperazone sodium & Cefobis & 1981 & I 127130 & & ND \\
\hline ceforanide & Precef & 1984 & ARMC 20 & 317 & ND \\
\hline cefoselis & Wincef & 1998 & ARMC 34 & 319 & ND \\
\hline cefotetan disodium & Yamatetan & 1984 & ARMC 20 & 317 & ND \\
\hline cefotiam $\mathrm{HCl}$ & Pansporin & 1981 & I 091106 & & ND \\
\hline cefozopran $\mathrm{HCl}$ & Firstcin & 1995 & ARMC 31 & 339 & ND \\
\hline cefpimizole & Ajicef & 1987 & ARMC 23 & 330 & ND \\
\hline cefpiramide sodium & Sepatren & 1985 & ARMC 21 & 325 & ND \\
\hline cefpirome sulfate & Cefrom & 1992 & ARMC 28 & 328 & ND \\
\hline cefpodoxime proxetil & Banan & 1989 & ARMC 25 & 310 & ND \\
\hline cefprozil & Cefzil & 1992 & ARMC 28 & 328 & ND \\
\hline cefsoludin sodium & Takesulin & 1981 & I 091108 & & ND \\
\hline ceftazidime & Fortam & 1983 & ARMC 19 & 316 & ND \\
\hline cefteram pivoxil & Tomiron & 1987 & ARMC 23 & 330 & ND \\
\hline ceftibuten & Seftem & 1992 & ARMC 28 & 329 & ND \\
\hline ceftizoxime sodium & Epocelin & 1982 & I 070260 & & ND \\
\hline ceftriaxone sodium & Rocephin & 1982 & I 091136 & & ND \\
\hline cefuroxime axetil & Zinnat & 1987 & ARMC 23 & 331 & ND \\
\hline cefuzonam sodium & Cosmosin & 1987 & ARMC 23 & 331 & ND \\
\hline clarithromycin & Klaricid & 1990 & ARMC 26 & 302 & ND \\
\hline dalfopristin & Synercid & 1999 & ARMC 35 & 338 & ND \\
\hline dirithromycin & Nortron & 1993 & ARMC 29 & 336 & ND \\
\hline doripenem & Finibax & 2005 & DNP 19 & 42 & ND \\
\hline ertapenem sodium & Invanz & 2002 & ARMC 38 & 353 & ND \\
\hline erythromycin acistrate & Erasis & 1988 & ARMC 24 & 301 & ND \\
\hline flomoxef sodium & Flumarin & 1988 & ARMC 24 & 302 & ND \\
\hline flurithromycin ethylsuccinate & Ritro & 1997 & ARMC 33 & 333 & ND \\
\hline fropenam & Farom & 1997 & ARMC 33 & 334 & ND \\
\hline imipenem/cilastatin & Zienam & 1985 & ARMC 21 & 328 & ND \\
\hline lenampicillin HCI & Varacillin & 1987 & ARMC 23 & 336 & ND \\
\hline loracarbef & Lorabid & 1992 & ARMC 28 & 333 & ND \\
\hline meropenem & Merrem & 1994 & ARMC 30 & 303 & ND \\
\hline moxalactam disodium & Shiomarin & 1982 & I 070301 & & ND \\
\hline panipenem/betamipron & Carbenin & 1994 & ARMC 30 & 305 & ND \\
\hline quinupristin & Synercid & 1999 & ARMC 35 & 338 & ND \\
\hline rifabutin & Mycobutin & 1992 & ARMC 28 & 335 & ND \\
\hline rifamixin & Normix & 1987 & ARMC 23 & 341 & ND \\
\hline rifapentine & Rifampin & 1988 & ARMC 24 & 310 & ND \\
\hline rifaximin & Rifacol & 1985 & ARMC 21 & 332 & ND \\
\hline rokitamycin & Ricamycin & 1986 & ARMC 22 & 325 & ND \\
\hline roxithromycin & Rulid & 1987 & ARMC 23 & 342 & ND \\
\hline sultamycillin tosylate & Unasyn & 1987 & ARMC 23 & 343 & ND \\
\hline tazobactam sodium & Tazocillin & 1992 & ARMC 28 & 336 & ND \\
\hline telithromycin & Ketek & 2001 & DNP 15 & 35 & ND \\
\hline temocillin disodium & Temopen & 1984 & ARMC 20 & 323 & ND \\
\hline tigecycline & Tygacil & 2005 & DNP 19 & 42 & ND \\
\hline
\end{tabular}


Table 2. Continued

\begin{tabular}{|c|c|c|c|c|c|}
\hline generic name & trade name & year introduced & reference & page & source \\
\hline balafloxacin & Q-Roxin & 2002 & ARMC 38 & 351 & $\mathrm{~S}$ \\
\hline ciprofloxacin & Ciprobay & 1986 & ARMC 22 & 318 & $\mathrm{~S}$ \\
\hline enoxacin & Flumark & 1986 & ARMC 22 & 320 & $\mathrm{~S}$ \\
\hline fleroxacin & Quinodis & 1992 & ARMC 28 & 331 & $\mathrm{~S}$ \\
\hline gatilfloxacin & Tequin & 1999 & ARMC 35 & 340 & $\mathrm{~S}$ \\
\hline gemifloxacin mesilate & Factive & 2003 & ARMC 40 & 458 & $\mathrm{~S}$ \\
\hline grepafloxacin & Vaxor & 1997 & DNP 11 & 23 & $\mathrm{~S}$ \\
\hline levofloxacin & Floxacin & 1993 & ARMC 29 & 340 & $\mathrm{~S}$ \\
\hline linezolid & Zyvox & 2000 & DNP 14 & 21 & $\mathrm{~S}$ \\
\hline lomefloxacin & Uniquin & 1989 & ARMC 25 & 315 & $\mathrm{~S}$ \\
\hline moxifloxacin $\mathrm{HCl}$ & Avelox & 1999 & ARMC 35 & 343 & $\mathrm{~S}$ \\
\hline nadifloxacin & Acuatim & 1993 & ARMC 29 & 340 & $\mathrm{~S}$ \\
\hline norfloxacin & Noroxin & 1983 & ARMC 19 & 322 & $\mathrm{~S}$ \\
\hline ofloxacin & Tarivid & 1985 & ARMC 21 & 331 & $\mathrm{~S}$ \\
\hline pazufloxacin & Pasil & 2002 & ARMC 38 & 364 & $\mathrm{~S}$ \\
\hline pefloxacin mesylate & Perflacine & 1985 & ARMC 21 & 331 & $\mathrm{~S}$ \\
\hline prulifloxacin & Sword & 2002 & ARMC 38 & 366 & $\mathrm{~S}$ \\
\hline rufloxacin hydrochloride & Qari & 1992 & ARMC 28 & 335 & $\mathrm{~S}$ \\
\hline sparfloxacin & Spara & 1993 & ARMC 29 & 345 & $\mathrm{~S}$ \\
\hline taurolidine & Taurolin & 1988 & I 107771 & & $\mathrm{~S}$ \\
\hline temafloxacin hydrochloride & Temac & 1991 & ARMC 27 & 334 & $\mathrm{~S}$ \\
\hline tosufloxacin & Ozex & 1990 & ARMC 26 & 310 & $\mathrm{~S}$ \\
\hline $\begin{array}{l}\text { trovafloxacin } \\
\text { mesylate }\end{array}$ & Trovan & 1998 & ARMC 34 & 332 & $\mathrm{~S}$ \\
\hline brodimoprin & Hyprim & 1993 & ARMC 29 & 333 & $\mathrm{~S} * / \mathrm{NM}$ \\
\hline ACWY meningoccal PS vaccine & Mencevax & 1981 & I 420128 & & $\mathrm{~V}$ \\
\hline $\mathrm{MCV}-4$ & Menactra & 2005 & DNP 19 & 43 & $\mathrm{~V}$ \\
\hline $\mathrm{h}$ influenzae $\mathrm{b}$ vaccine & Hibtitek & 1989 & DNP 03 & 24 & $\mathrm{~V}$ \\
\hline$h$ influenzae b vaccine & Prohibit & 1989 & DNP 03 & 24 & $\mathrm{~V}$ \\
\hline meningitis $\mathrm{b}$ vaccine & MeNZB & 2004 & DNP 18 & 29 & $\mathrm{~V}$ \\
\hline meningococcal vaccine & NeisVac-C & 2000 & DNP 14 & 22 & $\mathrm{~V}$ \\
\hline meningococcal vaccine & Menjugate & 2000 & DNP 14 & 22 & $\mathrm{~V}$ \\
\hline meningococcal vaccine & Menigetec & 1999 & DNP 14 & 22 & $\mathrm{~V}$ \\
\hline oral cholera vaccine & Orochol & 1994 & DNP 08 & 30 & $\mathrm{~V}$ \\
\hline pneumococcal vaccine & Prevnar & 2000 & DNP 14 & 22 & $\mathrm{~V}$ \\
\hline vi polysaccharide typhoid vaccine & Typherix & 1998 & DNP 12 & 35 & $\mathrm{~V}$ \\
\hline
\end{tabular}

Table 3. Antifungal Drugs from 01/1981 to 06/2006 Organized Alphabetically by Generic Name within Source

\begin{tabular}{|c|c|c|c|c|c|}
\hline generic name & trade name & year introduced & reference & page & source \\
\hline interferon gamma-n1 & OGamma100 & 1996 & DNP 10 & 13 & B \\
\hline anidulafungin & Eraxis & 2006 & I 194685 & & ND \\
\hline caspofungin acetate & Cancidas & 2001 & DNP 15 & 36 & ND \\
\hline micafungin sodium & Fungard & 2002 & ARMC 38 & 360 & ND \\
\hline amorolfine hydrochloride & Loceryl & 1991 & ARMC 27 & 322 & $\mathrm{~S}$ \\
\hline butoconazole & Femstat & 1986 & ARMC 22 & 318 & $\mathrm{~S}$ \\
\hline ciclopirox olamine & Loprox & 1982 & I 070449 & & $\mathrm{~S}$ \\
\hline cloconazole HCI & Pilzcin & 1986 & ARMC 22 & 318 & $\mathrm{~S}$ \\
\hline eberconazole & Ebernet & 2005 & DNP 19 & 42 & $\mathrm{~S}$ \\
\hline fenticonazole nitrate & Lomexin & 1987 & ARMC 23 & 334 & $\mathrm{~S}$ \\
\hline fluconazole & Diflucan & 1988 & ARMC 24 & 303 & $\mathrm{~S}$ \\
\hline flutrimazole & Micetal & 1995 & ARMC 31 & 343 & $\mathrm{~S}$ \\
\hline fosfluconazole & Prodif & 2003 & DNP 17 & 49 & $\mathrm{~S}$ \\
\hline itraconazole & Sporanox & 1988 & ARMC 24 & 305 & $\mathrm{~S}$ \\
\hline ketoconazole & Nizoral & 1981 & I 116505 & & $\mathrm{~S}$ \\
\hline lanoconazole & Astat & 1994 & ARMC 30 & 302 & $\mathrm{~S}$ \\
\hline luliconazole & Lulicon & 2005 & DNP 19 & 42 & $\mathrm{~S}$ \\
\hline naftifine $\mathrm{HCI}$ & Exoderil & 1984 & ARMC 20 & 321 & $\mathrm{~S}$ \\
\hline neticonazole HCI & Atolant & 1993 & ARMC 29 & 341 & $\mathrm{~S}$ \\
\hline oxiconazole nitrate & Oceral & 1983 & ARMC 19 & 322 & $\mathrm{~S}$ \\
\hline posaconazole & Noxafil & 2005 & DNP 19 & 42 & $\mathrm{~S}$ \\
\hline sertaconazole nitrate & Dermofix & 1992 & ARMC 28 & 336 & $\mathrm{~S}$ \\
\hline sulconazole nitrate & Exelderm & 1985 & ARMC 21 & 332 & $\mathrm{~S}$ \\
\hline terconazole & Gyno-Terazol & 1983 & ARMC 19 & 324 & $\mathrm{~S}$ \\
\hline tioconazole & Trosyl & 1983 & ARMC 19 & 324 & $\mathrm{~S}$ \\
\hline voriconazole & Vfend & 2002 & ARMC 38 & 370 & $\mathrm{~S}$ \\
\hline butenafine hydrochloride & Mentax & 1992 & ARMC 28 & 327 & S/NM \\
\hline liranaftate & Zefnart & 2000 & DNP 14 & 21 & S/NM \\
\hline terbinafine hydrochloride & Lamisil & 1991 & ARMC 27 & 334 & S/NM \\
\hline
\end{tabular}

Overview of Results. The data that we have analyzed in a variety of ways are presented as a series of bar graphs and pie charts and two major tables in order to establish the overall pictures and then are further subdivided into some major therapeutic areas using a tabular format. Except where noted, the time frame covered was 01/1981-06/2006:

- New Approved Drugs: With all source categories (Figure 1)

- New Approved Drugs: By source/year (Figure 2) 
Table 4. Antiviral Drugs from 01/1981 to 06/2006 Organized Alphabetically by Generic Name within Source

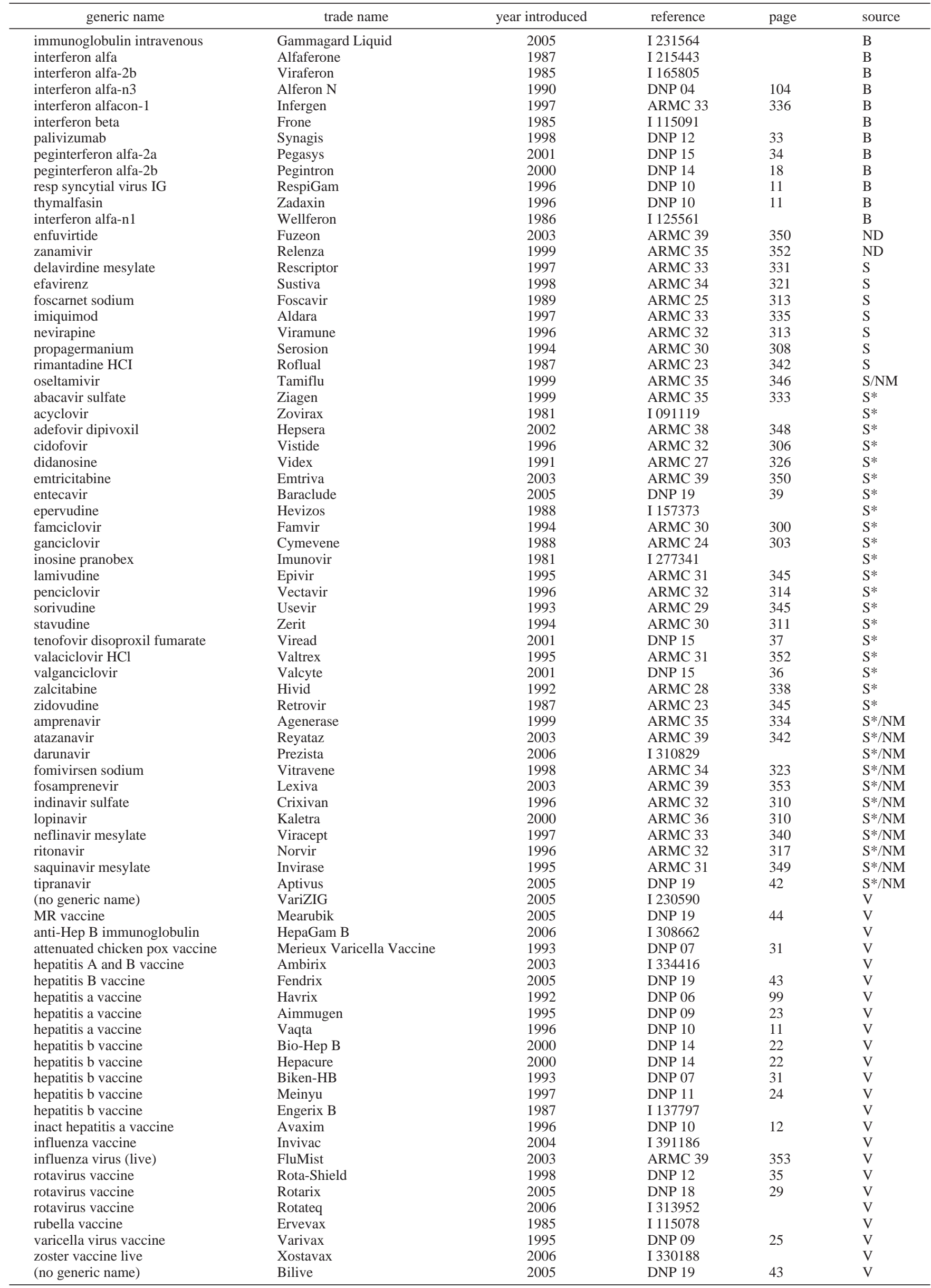


Table 5. Antiparasitic Drugs from 01/1981 to 06/2006 Organized Alphabetically by Generic Name within Source

\begin{tabular}{|c|c|c|c|c|c|}
\hline generic name & trade name & year introduced & reference & page & source \\
\hline artemisinin & Artemisin & 1987 & ARMC 23 & 327 & $\mathrm{~N}$ \\
\hline ivermectin & Mectizan & 1987 & ARMC 23 & 336 & $\mathrm{~N}$ \\
\hline arteether & Artemotil & 2000 & DNP 14 & 22 & ND \\
\hline artemether & Artemetheri & 1987 & I 090712 & & ND \\
\hline artenusate & Arinate & 1987 & I 091299 & & ND \\
\hline eflornithine $\mathrm{HCl}$ & Ornidyl & 1990 & DNP 04 & 104 & ND \\
\hline mefloquine $\mathrm{HCI}$ & Fansimef & 1985 & ARMC 21 & 329 & ND \\
\hline albendazole & Eskazole & 1982 & I 129625 & & $\mathrm{~S}$ \\
\hline halofantrine & Halfan & 1988 & ARMC 24 & 304 & $\mathrm{~S}$ \\
\hline lumefantrine & no trade name & 1987 & I 269095 & & $\mathrm{~S}$ \\
\hline quinfamide & Amenox & 1984 & ARMC 20 & 322 & $\mathrm{~S}$ \\
\hline atovaquone & Mepron & 1992 & ARMC 28 & 326 & $\mathrm{~S}^{*}$ \\
\hline bulaquine/chloroquine & Aablaquin & 2000 & DNP 14 & 22 & $\mathrm{~S}^{*}$ \\
\hline trichomonas vaccine & Gynatren & 1986 & I 125543 & & $\mathrm{~V}$ \\
\hline
\end{tabular}

Table 6. All Antiinfective (Antibacterial, Fungal, Parasitic, and Viral) Drugs $(N=230)$

\begin{tabular}{llllllllll}
\hline \multicolumn{1}{c}{ indication } & total & $\mathrm{B}$ & $\mathrm{N}$ & $\mathrm{ND}$ & $\mathrm{S}$ & $\mathrm{S} / \mathrm{NM}$ & $\mathrm{S} *$ & $\mathrm{~S} * / \mathrm{NM}$ & $\mathrm{V}$ \\
\hline antibacterial & 109 & & 10 & 64 & 23 & & & 1 & 11 \\
antifungal & 29 & 1 & & 3 & 22 & 3 & & & \\
antiparasitic & 14 & & 2 & 5 & 4 & & 2 & & 1 \\
antiviral & 78 & 12 & & 2 & 7 & 1 & 20 & 12 & 25 \\
total & 230 & 13 & 12 & 74 & 56 & 4 & 22 & 12 & 37 \\
percentage & 100.0 & 5.7 & 5.2 & 32.3 & 24.5 & 2.2 & 9.6 & 4.8 & 15.7 \\
\hline
\end{tabular}

Table 7. Small Molecule Antiinfective (Antibacterial, Fungal, Parasitic, and Viral) Drugs $(N=180)$

\begin{tabular}{lllllllc}
\hline \multicolumn{1}{c}{ indication } & total & $\mathrm{N}$ & $\mathrm{ND}$ & $\mathrm{S}$ & $\mathrm{S} / \mathrm{NM}$ & $\mathrm{S} *$ & $\mathrm{~S} * / \mathrm{NM}$ \\
\hline antibacterial & 98 & 10 & 64 & 23 & & & 1 \\
antifungal & 29 & & 3 & 22 & 3 & & \\
antiparasitic & 13 & 2 & 5 & 4 & & 2 & \\
antiviral & 41 & & 2 & 7 & 1 & 20 & 12 \\
total & 180 & 12 & 74 & 56 & 4 & 22 & 11 \\
percentage & 100.0 & 6.7 & 41.1 & 31.1 & 2.8 & 12.2 & 6.1 \\
\hline
\end{tabular}

- Sources of all NCEs: Where four or more drugs were approved per medical indication (Table 1) 3)

- Sources of Small Molecule NCEs: All subdivisions (Figure

- Sources of Small Molecule NCEs: By source/year (Figure 4)

- Antibacterial Drugs: Generic and trade names, year, reference, and source (Table 2)

- Antifungal Drugs: Generic and trade names, year, reference, and source (Table 3)

- Antiviral Drugs: Generic and trade names, year, reference, and source (Table 4)

- Antiparasitic Drugs: Generic and trade names, year, reference, and source (Table 5)

- Antiinfective Drugs: All molecules, source, and numbers (Table 6)

- Antiinfective Drugs: Small molecules, source, and numbers (Table 7)

- Anticancer Drugs: Generic and trade names, year, reference, and source (Table 8)

- All Anticancer Drugs: Generic names, reference, and source (Figures 5-7; and (1940s-06/2006) Table 9)

- Antidiabetic Drugs: Generic and trade names, year, reference, and source (Table 10)

The extensive data sets shown in the figures and tables referred to above highlight the continuing role that natural products and structures derived from or related to natural products from all sources have played and continue to play in the development of the current therapeutic armamentarium of the physician. Inspection of the data shows this continued important role for natural products in spite of the current low level of natural products-based drug discovery programs in major pharmaceutical houses.

Inspection of the rate of NCE approvals as shown in Figure 2 demonstrates that the natural products field is still producing or is involved in $\sim 50 \%$ of all small molecules in the years 2000-2006 and that a significant number of NCEs are biologicals or vaccines ( 83 of 264 , or $31.4 \%$ ). This is so in spite of many years of work by the pharmaceutical industry devoted to high-throughput screening of predominately combinatorial chemistry products and that the time period chosen should have provided a sufficient time span for combinatorial chemistry work from the late 1980s onward to have produced approved NCEs.

Overall, of the 1184 NCEs covering all diseases/countries/sources in the years 01/1981-06/2006, and using the "NM" classifications introduced in our 2003 review, ${ }^{1,2} 30 \%$ were synthetic in origin, thus demonstrating the influence of "other than formal synthetics" on drug discovery and approval (Figure 1).

Inspection of Table 1 demonstrates that, overall, the major disease areas that have been investigated (in terms of numbers of drugs approved) in the pharmaceutical industry continue to be infectious diseases (microbial, parasitic, and viral), cancer, antihypertensives, and antiinflammatory indications, all with over 50 approved drug therapies. It should be noted, however, that numbers of approved drugs/disease do not correlate with the "value" as measured by sales, since the best selling drug of all is atorvastin, a hypocholesterolemic descended directly from a natural product, which sold over $\$ 11$ billion in 2004 and is at or above this level even today.

The major category by far is that of antiinfectives including antiviral vaccines, with $230(22.8 \%)$ of the total (1010 for indications $\geq 4$ ) falling into this one major human disease area. On further analyses (Tables 6 and 7), the influence of biologicals and vaccines in this disease complex is such that only a little over $30 \%$ are synthetic in origin. If one considers only small molecules (reducing the total by 50 to 180; Table 10), then the synthetic figure goes up to $31.1 \%$, marginally greater than in our previous report. ${ }^{2}$ As reported previously, ${ }^{1,2}$ these synthetic drugs actually tend to be of two basic chemotypes, the azole-based antifungals and the quinolone-based antibacterials.

Four small molecule drugs were approved in the antibacterial area from 01/2003 to 06/2006. These included daptomycin (N, 5) from Cubist, a lipopeptide whose biosynthetic cluster has been successfully cloned and expressed by investigators associated with Cubist. ${ }^{71}$ Wyeth had their modified tetracycline derivative, tigecycline, approved (ND, 6), a drug designed to overcome the tet resistance pump in pathogenic bacteria, and another carbapenem (ND) and a quinolone (S) were also approved in this time frame. In the antifungal area, of the five drugs approved, four were azoles (S) and the echinocandin derivative, anidulofungin (ND), was approved for use in the U.S. in early 2006. In the antiviral area, seven drugs were approved for HIV treatment (1 ND, $1 \mathrm{~S}^{*}, 5 \mathrm{~S}^{*} /$ $\mathrm{NM}$ ). It is interesting that the one ND, enfuvirtide, though listed in most literature as a synthetic, is actually the "end-capped" 36- 
Table 8. Anticancer Drugs from 01/1981-06/2006 Organized Alphabetically by Generic Name within Source

\begin{tabular}{|c|c|c|c|c|c|}
\hline generic name & trade name & year introduced & reference & page & source \\
\hline H-101 & none given ${ }^{a}$ & 2005 & DNP 19 & 46 & B \\
\hline alemtuzumab & Campath & 2001 & DNP 15 & 38 & B \\
\hline bevacizumab & Avastin & 2004 & ARMC 40 & 450 & B \\
\hline celmoleukin & Celeuk & 1992 & DNP 06 & 102 & B \\
\hline cetuximab & Erbitux & 2003 & ARMC 39 & 346 & B \\
\hline denileukin diftitox & Ontak & 1999 & ARMC 35 & 338 & B \\
\hline ibritumomab & Zevalin & 2002 & ARMC 38 & 359 & B \\
\hline interferon alfa2a & Roferon-A & 1986 & I 204503 & & B \\
\hline interferon, gamma-1a & Biogamma & 1992 & ARMC 28 & 332 & B \\
\hline interleukin-2 & Proleukin & 1989 & ARMC 25 & 314 & B \\
\hline mobenakin & Octin & 1999 & ARMC 35 & 345 & B \\
\hline pegaspargase & Oncaspar & 1994 & ARMC 30 & 306 & B \\
\hline rituximab & Rituxan & 1997 & DNP 11 & 25 & B \\
\hline tasonermin & Beromun & 1999 & ARMC 35 & 349 & B \\
\hline teceleukin & Imumace & 1992 & DNP 06 & 102 & B \\
\hline tositumomab & Bexxar & 2003 & ARMC 39 & 364 & B \\
\hline trastuzumab & Herceptin & 1998 & DNP 12 & 35 & B \\
\hline aclarubicin & Aclacin & 1981 & I 090013 & & $\mathrm{~N}$ \\
\hline angiotensin II & Delivert & 1994 & ARMC 30 & 296 & $\mathrm{~N}$ \\
\hline arglabin & none given ${ }^{a}$ & 1999 & ARMC 35 & 335 & $\mathrm{~N}$ \\
\hline masoprocol & Actinex & 1992 & ARMC 28 & 333 & $\mathrm{~N}$ \\
\hline paclitaxel & Taxol & 1993 & ARMC 29 & 342 & $\mathrm{~N}$ \\
\hline paclitaxel nanoparticles & Abraxane & 2005 & DNP 19 & 45 & $\mathrm{~N}$ \\
\hline pentostatin & Nipent & 1992 & ARMC 28 & 334 & $\mathrm{~N}$ \\
\hline peplomycin & Pepleo & 1981 & I 090889 & & $\mathrm{~N}$ \\
\hline solamargines & Curaderm & 1989 & DNP 03 & 25 & $\mathrm{~N}$ \\
\hline alitretinoin & Panretin & 1999 & ARMC 35 & 333 & ND \\
\hline amrubicin $\mathrm{HCl}$ & Calsed & 2002 & ARMC 38 & 349 & ND \\
\hline belotecan hydrochloride & Camtobell & 2004 & ARMC 40 & 449 & ND \\
\hline cladribine & Leustatin & 1993 & ARMC 29 & 335 & ND \\
\hline cytarabine ocfosfate & Starsaid & 1993 & ARMC 29 & 335 & ND \\
\hline docetaxel & Taxotere & 1995 & ARMC 31 & 341 & ND \\
\hline elliptinium acetate & Celiptium & 1983 & I 091123 & & ND \\
\hline epirubicin HCI & Farmorubicin & 1984 & ARMC 20 & 318 & ND \\
\hline etoposide phosphate ${ }^{b}$ & Etopophos & 1996 & DNP 10 & 13 & ND \\
\hline exemestane & Aromasin & 1999 & DNP 13 & 46 & ND \\
\hline formestane & Lentaron & 1993 & ARMC 29 & 337 & ND \\
\hline fulvestrant & Faslodex & 2002 & ARMC 38 & 357 & ND \\
\hline gemtuzumab ozogamicin & Mylotarg & 2000 & DNP 14 & 23 & ND \\
\hline hexyl aminolevulinate & Hexvix & 2004 & I 300211 & & ND \\
\hline idarubicin hydrochloride & Zavedos & 1990 & ARMC 26 & 303 & ND \\
\hline irinotecan hydrochloride & Campto & 1994 & ARMC 30 & 301 & ND \\
\hline miltefosine & Miltex & 1993 & ARMC 29 & 340 & ND \\
\hline pirarubicin & Pinorubicin & 1988 & ARMC 24 & 309 & ND \\
\hline talaporfin sodium & Laserphyrin & 2004 & ARMC 40 & 469 & ND \\
\hline topotecan $\mathrm{HCl}$ & Hycamptin & 1996 & ARMC 32 & 320 & ND \\
\hline triptorelin & Decapeptyl & 1986 & I 090485 & & ND \\
\hline valrubicin & Valstar & 1999 & ARMC 35 & 350 & ND \\
\hline vapreotide acetate & Docrised & 2004 & I 135014 & & ND \\
\hline vinorelbine & Navelbine & 1989 & ARMC 25 & 320 & ND \\
\hline zinostatin stimalamer & Smancs & 1994 & ARMC 30 & 313 & ND \\
\hline aminoglutethimide & Cytadren & 1981 & I 070408 & & $\mathrm{~S}$ \\
\hline amsacrine & Amsakrin & 1987 & ARMC 23 & 327 & $\mathrm{~S}$ \\
\hline arsenic trioxide & Trisenox & 2000 & DNP 14 & 23 & $\mathrm{~S}$ \\
\hline bisantrene hydrochloride & Zantrene & 1990 & ARMC 26 & 300 & $\mathrm{~S}$ \\
\hline carboplatin & Paraplatin & 1986 & ARMC 22 & 318 & $\mathrm{~S}$ \\
\hline flutamide & Drogenil & 1983 & ARMC 19 & 318 & $\mathrm{~S}$ \\
\hline fotemustine & Muphoran & 1989 & ARMC 25 & 313 & $\mathrm{~S}$ \\
\hline heptaplatin/SK-2053R & Sunpla & 1999 & ARMC 35 & 348 & $\mathrm{~S}$ \\
\hline lobaplatin & Lobaplatin & 1998 & DNP 12 & 35 & $\mathrm{~S}$ \\
\hline lonidamine & Doridamina & 1987 & ARMC 23 & 337 & $\mathrm{~S}$ \\
\hline nedaplatin & Aqupla & 1995 & ARMC 31 & 347 & $\mathrm{~S}$ \\
\hline nilutamide & Anadron & 1987 & ARMC 23 & 338 & $\mathrm{~S}$ \\
\hline oxaliplatin & Eloxatin & 1996 & ARMC 32 & 313 & $\mathrm{~S}$ \\
\hline porfimer sodium & Photofrin & 1993 & ARMC 29 & 343 & $\mathrm{~S}$ \\
\hline ranimustine & Cymerine & 1987 & ARMC 23 & 341 & $\mathrm{~S}$ \\
\hline sobuzoxane & Parazolin & 1994 & ARMC 30 & 310 & $\mathrm{~S}$ \\
\hline sorafenib & Nexavar & 2005 & DNP 19 & 45 & $\mathrm{~S}$ \\
\hline anastrozole & Arimidex & 1995 & ARMC 31 & 338 & $\mathrm{~S} / \mathrm{NM}$ \\
\hline bicalutamide & Casodex & 1995 & ARMC 31 & 338 & $\mathrm{~S} / \mathrm{NM}$ \\
\hline bortezomib & Velcade & 2003 & ARMC 39 & 345 & $\mathrm{~S} / \mathrm{NM}$ \\
\hline camostat mesylate & Foipan & 1985 & ARMC 21 & 325 & S/NM \\
\hline erlotinib hydrochloride & Tarceva & 2004 & ARMC 40 & 454 & S/NM \\
\hline fadrozole $\mathrm{HCl}$ & Afema & 1995 & ARMC 31 & 342 & $\mathrm{~S} / \mathrm{NM}$ \\
\hline gefitinib & Iressa & 2002 & ARMC 38 & 358 & S/NM \\
\hline imatinib mesilate & Gleevec & 2001 & DNP 15 & 38 & $\mathrm{~S} / \mathrm{NM}$ \\
\hline letrazole & Femara & 1996 & ARMC 32 & 311 & S/NM \\
\hline
\end{tabular}


Table 8. Continued

\begin{tabular}{|c|c|c|c|c|c|}
\hline generic name & trade name & year introduced & reference & page & source \\
\hline sunitinib malate & Sutent & 2006 & I 309144 & & $\mathrm{~S} / \mathrm{NM}$ \\
\hline temoporfin & Foscan & 2002 & I 158118 & & $\mathrm{~S} / \mathrm{NM}$ \\
\hline toremifene & Fareston & 1989 & ARMC 25 & 319 & $\mathrm{~S} / \mathrm{NM}$ \\
\hline zoledronic acid & Zometa & 2000 & DNP 14 & 24 & $\mathrm{~S}$ \\
\hline azacytidine & Vidaza & 2004 & ARMC 40 & 447 & $\mathrm{~S}^{*}$ \\
\hline capecitabine & Xeloda & 1998 & ARMC 34 & 319 & $\mathrm{~S}^{*}$ \\
\hline carmofur & Mifurol & 1981 & I 091100 & & $\mathrm{~S}^{*}$ \\
\hline clofarabine & Clolar & 2005 & DNP 19 & 44 & $\mathrm{~S}^{*}$ \\
\hline decitabine & Dacogen & 2006 & I 125366 & & $\mathrm{~S}^{*}$ \\
\hline doxifluridine & Furtulon & 1987 & ARMC 23 & 332 & $\mathrm{~S}^{*}$ \\
\hline enocitabine & Sunrabin & 1983 & ARMC 19 & 318 & $\mathrm{~S}^{*}$ \\
\hline fludarabine phosphate & Fludara & 1991 & ARMC 27 & 327 & $\mathrm{~S}^{*}$ \\
\hline gemcitabine $\mathrm{HCl}$ & Gemzar & 1995 & ARMC 31 & 344 & $\mathrm{~S} *$ \\
\hline mitoxantrone $\mathrm{HCI}$ & Novantrone & 1984 & ARMC 20 & 321 & $\mathrm{~S}^{*}$ \\
\hline nelarabine & Arranon & 2005 & DNP 19 & 45 & $\mathrm{~S}^{*}$ \\
\hline abarelix & Plenaxis & 2004 & ARMC 40 & 446 & $\mathrm{~S} * / \mathrm{NM}$ \\
\hline bexarotene & Targretine & 2000 & DNP 14 & 23 & $\mathrm{~S} * / \mathrm{NM}$ \\
\hline pemetrexed disodium & Alimta & 2004 & ARMC 40 & 463 & $\mathrm{~S} * / \mathrm{NM}$ \\
\hline raltitrexed & Tomudex & 1996 & ARMC 32 & 315 & $\mathrm{~S} * / \mathrm{NM}$ \\
\hline tamibarotene & Amnoid & 2005 & DNP 19 & 45 & $\mathrm{~S} * / \mathrm{NM}$ \\
\hline temozolomide & Temodal & 1999 & ARMC 35 & 350 & $\mathrm{~S} * / \mathrm{NM}$ \\
\hline bcg live & TheraCys & 1990 & DNP 04 & 104 & V \\
\hline melanoma theraccine & Melacine & 2001 & DNP 15 & 38 & $\mathrm{~V}$ \\
\hline
\end{tabular}

${ }^{a}$ No trade name given in the original report nor in the Prous Integrity database. ${ }^{b}$ A prodrug of etoposide.

residue peptide that corresponds to residues 643-678 of the HIV-1 transmembrane protein gp41 and blocks viral fusion with the cell. ${ }^{72}$ In addition to this novel mechanism, four new HIV protease inhibitors were approved; all were peptidomimetics imitating the peptide substrate, and the latest one, darunavir (7), actually has the hydroxyethyl isostere that was first identified in the microbial aspartic protease inhibitor pepstatin and incorporated in the base structure of crixivan (see discussion by Yang et al. ${ }^{73}$ ).
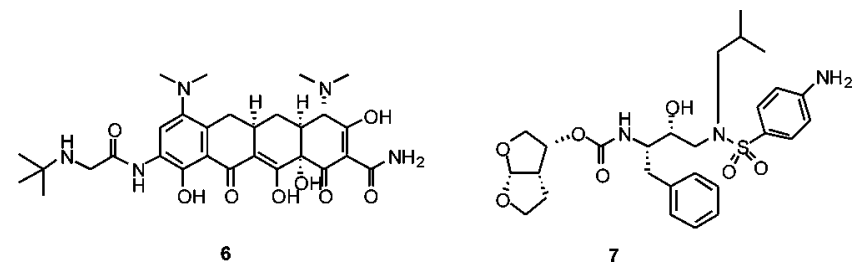

It should be noted that the percentages used in the following overall analyses do not always agree with those in the later tables, as all sources, which include $\mathrm{B}$ and $\mathrm{V}$ categorized drugs, and all indications are included in the percentage figures used in the analyses. Much fuller details are given in the Supporting Information in the form of an Excel XP spreadsheet.

As we reported in our earlier analyses, ${ }^{1,2}$ there are still significant therapeutic classes where the available drugs are totally synthetic at the present time. These include antihistamines, diuretics, and hypnotics for indications with four or more approved drugs (cf. Table 1). There are a substantial number of indications where there are three or less drugs that are also totally synthetic. Because of our introduction of the "NM" subcategory, indications such as antidepressants and cardiotonics now have substantial numbers that, although formally "S", now fall into the "S/NM" subcategory.

From inspection of Tables $1-4$ and 8 and the Excel XP spreadsheet, the following points can be made in addition to the digest on antiinfectives given in Tables 6 and 7. In the antibacterial area (Table 2), as found previously, the vast majority of the 98 small molecule NCEs are N $(10 ; 10.2 \%)$, ND $(64 ; 65.3 \%)$, or S*/ NM $(1 ; 1 \%)$, amounting to 75 in total, or $76.5 \%$ of the whole, with the remainder $(\mathrm{S})$ being predominately quinolones. In the antifungal area (Table 3$)$, the roles of the small molecules $(n=28)$ are reversed, with the great majority being $\mathrm{S}(22 ; 78.6 \%)$ and S/NM (3; 10.7\%), with the remainder being ND $(3 ; 10.7 \%)$.

In the antiviral area (Table 4), the situation is somewhat different, with a large number of vaccines $(n=25)$ now added to this category. If we consider only small molecules, the anti-HIV drugs being approved are based mainly on nucleoside structures $\left(\mathrm{S}^{*}\right)$ or on peptidomimetics ( $\mathrm{S}^{*}$ and $\mathrm{S} / \mathrm{NM}$ ), and drugs against other viral diseases also fall into these categories. Thus, one can see that of the 42 small molecule approved antiviral agents, the relevant figures are ND $(2 ; 4.8 \%), \mathrm{S}^{*}$ and $\mathrm{S}^{*} / \mathrm{NM}$ categories $(32 ; 76.2 \%)$, with the remainder falling into either $\mathrm{S}(7 ; 16.7 \%)$ or S/NM $(1 ; 2.4 \%)$.

We have also identified the antiparasitic drugs that have been approved over the years (Table 5) and point out that of the 14 small molecule drugs, only four are synthetic $(28.5 \%)$ and of the rest, three are artemisinin derivatives. What is of interest with this base structure is that, in addition to their known antimalarial activities, compounds based on this structure are demonstrating activity as antitumor agents. ${ }^{74}$

With anticancer drugs (Table 8), where in the time frame covered (01/1981-06/2006) there were 100 NCEs in toto, the number of nonbiologicals was $81(81 \%)$. These small molecules could be divided as follows (using $81=100 \%)$ into $\mathrm{N}(9 ; 11.1 \%)$, ND $(25$; $30.9 \%), \mathrm{S}(18 ; 22.2 \%), \mathrm{S} / \mathrm{NM}(12 ; 14.8 \%), \mathrm{S} *(11 ; 13.6 \%)$, and $\mathrm{S} * / \mathrm{NM}(6 ; 7.4 \%)$. Thus, using our criteria, only $22.2 \%$ of the total number of anticancer drugs were classifiable into the $\mathrm{S}$ (synthetic) category. Expressed as a proportion of the nonbiologicals/vaccines, then 63 of $81(77.8 \%)$ were either natural products per se or were based thereon, or mimicked natural products in one form or another.

In this current review, we have continued as in our previous contribution $(2003)^{2}$ to reassess the influence of natural products and their mimics as leads to anticancer drugs. By using data from the FDA listings of antitumor drugs, coupled with our previous data sources and with help from Japanese colleagues, we have been able to identify the years in which all but 18 of the 175 drugs we have listed in Table 9 were approved. We have identified these other 18 agents by inspection of three time-relevant textbooks on antitumor treatment, ${ }^{51,75,76}$ and these were added to the overall listings using the lead authors' names as the source citation.

Inspection of Figures 5-7 and Table 9 shows that, over the whole category of anticancer drugs effectively available to the West and Japan, the 175 available agents can be categorized as follows: B (18; $10 \%), \mathrm{N}(25 ; 14 \%)$, ND (48; 28\%), S (42; 24\%), S/NM (14; $8 \%), \mathrm{S}^{*}(20 ; 11 \%), \mathrm{S} * \mathrm{NM}(6 ; 4 \%)$, and $\mathrm{V}(2 ; 1 \%)$. If one removes the biologicals and vaccines, reducing the overall number to 155 $(100 \%)$, the number of naturally inspired agents (i.e., N, ND, S/NM, $\left.\mathrm{S}^{*}, \mathrm{~S}^{*} / \mathrm{NM}\right)$ is $113(72.9 \%)$. It should be noted that these 155 agents do not include some of the earlier drugs that were really immuno- 
Table 9. All Anticancer Drugs (1940s-07/2006) $)^{a}$ Organized Alphabetically by Generic Name within Source

\begin{tabular}{|c|c|c|c|c|}
\hline generic name & $\begin{array}{c}\text { year } \\
\text { introduced }\end{array}$ & reference & page & source \\
\hline H-101 & 2005 & DNP 19 & 46 & $\mathrm{~B}$ \\
\hline aldesleukin & 1992 & ARMC 25 & 314 & B \\
\hline alemtuzumab & 2001 & DNP 15 & 38 & $\mathrm{~B}$ \\
\hline bevacizumab & 2004 & ARMC 40 & 450 & $\mathrm{~B}$ \\
\hline celmoleukin & 1992 & DNP 06 & 102 & B \\
\hline cetuximab & 2003 & ARMC 39 & 346 & $\mathrm{~B}$ \\
\hline denileukin diftitox & 1999 & ARMC 35 & 338 & $\mathrm{~B}$ \\
\hline interferon alfa2a & 1986 & I 204503 & & $\mathrm{~B}$ \\
\hline interferon alfa2b & 1986 & I 165805 & & B \\
\hline interferon, gamma-1a & 1992 & ARMC 28 & 332 & $\mathrm{~B}$ \\
\hline interleukin-2 & 1989 & ARMC 25 & 314 & $\mathrm{~B}$ \\
\hline mobenakin & 1999 & ARMC 35 & 345 & $\mathrm{~B}$ \\
\hline pegaspargase & 1994 & ARMC 30 & 306 & $\mathrm{~B}$ \\
\hline rituximab & 1997 & DNP 11 & 25 & B \\
\hline tasonermin & 1999 & ARMC 35 & 349 & $\mathrm{~B}$ \\
\hline teceleukin & 1992 & DNP 06 & 102 & $\mathrm{~B}$ \\
\hline tositumomab & 2003 & ARMC 39 & 364 & $\mathrm{~B}$ \\
\hline trastuzumab & 1998 & DNP 12 & 35 & $\mathrm{~B}$ \\
\hline aclarubicin & 1981 & I 090013 & & $\mathrm{~N}$ \\
\hline actinomycin D & 1964 & FDA & & $\mathrm{N}$ \\
\hline angiotensin II & 1994 & ARMC 30 & 296 & $\mathrm{~N}$ \\
\hline arglabin & 1999 & ARMC 35 & 335 & $\mathrm{~N}$ \\
\hline asparaginase & 1969 & FDA & & $\mathrm{N}$ \\
\hline bleomycin & 1966 & FDA & & $\mathrm{N}$ \\
\hline carzinophilin & 1954 & Japan Antibiotics & & $\mathrm{N}$ \\
\hline chromomycin A3 & 1961 & Japan Antibiotics & & $\mathrm{N}$ \\
\hline daunomycin & 1967 & FDA & & $\mathrm{N}$ \\
\hline doxorubicin & 1966 & FDA & & $\mathrm{N}$ \\
\hline leucovorin & 1950 & FDA & & $\mathrm{N}$ \\
\hline masoprocol & 1992 & ARMC 28 & 333 & $\mathrm{~N}$ \\
\hline mithramycin & 1961 & FDA & & $\mathrm{N}$ \\
\hline mitomycin C & 1956 & FDA & & $\mathrm{N}$ \\
\hline neocarzinostatin & 1976 & Japan Antibiotics & & $\mathrm{N}$ \\
\hline paclitaxel & 1993 & ARMC 29 & 342 & $\mathrm{~N}$ \\
\hline palictaxel nanoparticles & 2005 & DNP 19 & 45 & $\mathrm{~N}$ \\
\hline pentostatin & 1992 & ARMC 28 & 334 & $\mathrm{~N}$ \\
\hline peplomycin & 1981 & I 090889 & & $\mathrm{~N}$ \\
\hline sarkomycin & 1954 & FDA & & $\mathrm{N}$ \\
\hline solamargine (aka BEC) & 1987 & DNP 03 & 25 & $\mathrm{~N}$ \\
\hline streptozocin & pre-1977 & & & $\mathrm{N}$ \\
\hline testosterone & pre-1970 & & & $\mathrm{N}$ \\
\hline vinblastine & 1965 & FDA & & $\mathrm{N}$ \\
\hline vincristine & 1963 & FDA & & $\mathrm{N}$ \\
\hline alitretinoin & 1999 & ARMC 35 & 333 & ND \\
\hline amrubicin $\mathrm{HCl}$ & 2002 & ARMC 38 & 349 & ND \\
\hline belotecan hydrocholoride & 2004 & ARMC 40 & 449 & ND \\
\hline calusterone & 1973 & FDA & & ND \\
\hline cladribine & 1993 & ARMC 29 & 335 & ND \\
\hline cytarabine ocfosfate & 1993 & ARMC 29 & 335 & ND \\
\hline dexamethasone & 1958 & FDA & & ND \\
\hline docetaxel & 1995 & ARMC 31 & 341 & ND \\
\hline dromostanolone & 1961 & FDA & & ND \\
\hline elliptinium acetate & 1983 & I 091123 & & ND \\
\hline epirubicin HCI & 1984 & ARMC 20 & 318 & ND \\
\hline estramustine & 1980 & FDA & & ND \\
\hline ethinyl estradiol & pre-1970 & & & ND \\
\hline etoposide & 1980 & FDA & & ND \\
\hline exemestane & 1999 & DNP 13 & 46 & ND \\
\hline fluoxymesterone & pre-1970 & & & ND \\
\hline formestane & 1993 & ARMC 29 & 337 & ND \\
\hline fosfestrol & pre-1977 & & & ND \\
\hline fulvestrant & 2002 & ARMC 38 & 357 & ND \\
\hline gemtuzumab ozogamicin & 2000 & DNP 14 & 23 & ND \\
\hline goserelin acetate & 1987 & ARMC 23 & 336 & ND \\
\hline hexyl aminolevulinate & 2004 & I 300211 & & ND \\
\hline histrelin & 2004 & I 109865 & & ND \\
\hline hydroxyprogesterone & pre-1970 & & & ND \\
\hline idarubicin hydrochloride & 1990 & ARMC 26 & 303 & ND \\
\hline irinotecan hydrochloride & 1994 & ARMC 30 & 301 & ND \\
\hline leuprolide & 1984 & ARMC 20 & 319 & ND \\
\hline medroxyprogesterone acetate & 1958 & FDA & & ND \\
\hline megesterol acetate & 1971 & FDA & & ND \\
\hline methylprednisolone & 1955 & FDA & & ND \\
\hline methyltestosterone & 1974 & FDA & & ND \\
\hline miltefosine & 1993 & ARMC 29 & 340 & ND \\
\hline mitobronitol & 1979 & FDA & & ND \\
\hline nadrolone phenylpropionate & 1959 & FDA & & ND \\
\hline
\end{tabular}


Table 9. Continued

\begin{tabular}{|c|c|c|c|c|}
\hline generic name & year introduced & reference & page & source \\
\hline norethindrone acetate & pre-1977 & & & ND \\
\hline pirarubicin & 1988 & ARMC 24 & 309 & ND \\
\hline prednisolone & pre-1977 & & & ND \\
\hline prednisone & pre-1970 & & & ND \\
\hline teniposide & 1967 & FDA & & ND \\
\hline testolactone & 1969 & FDA & & ND \\
\hline topotecan $\mathrm{HCl}$ & 1996 & ARMC 32 & 320 & ND \\
\hline triamcinolone & 1958 & FDA & & ND \\
\hline triptorelin & 1986 & I 090485 & & ND \\
\hline valrubicin & 1999 & ARMC 35 & 350 & ND \\
\hline vapreotide acetate & 2003 & I 135014 & & ND \\
\hline vindesine & 1979 & FDA & & ND \\
\hline vinorelbine & 1989 & ARMC 25 & 320 & ND \\
\hline zinostatin stimalamer & 1994 & ARMC 30 & 313 & ND \\
\hline amsacrine & 1987 & ARMC 23 & 327 & S \\
\hline arsenic trioxide & 2000 & DNP 14 & 23 & $\mathrm{~S}$ \\
\hline bisantrene hydrochloride & 1990 & ARMC 26 & 300 & $\mathrm{~S}$ \\
\hline busulfan & 1954 & FDA & & $\mathrm{S}$ \\
\hline carboplatin & 1986 & ARMC 22 & 318 & $\mathrm{~S}$ \\
\hline carmustine (BCNU) & 1977 & FDA & & $\mathrm{S}$ \\
\hline chlorambucil & 1956 & FDA & & $\mathrm{S}$ \\
\hline chlortrianisene & pre-1981 & BOYD & & $\mathrm{S}$ \\
\hline cis-diamminedichloroplatinum & 1979 & FDA & & $\mathrm{S}$ \\
\hline cyclophosphamide & 1957 & FDA & & $\mathrm{S}$ \\
\hline dacarbazine & 1975 & FDA & & $\mathrm{S}$ \\
\hline diethylstilbestrol & pre-1970 & & & $\mathrm{S}$ \\
\hline flutamide & 1983 & ARMC 19 & 318 & $\mathrm{~S}$ \\
\hline fotemustine & 1989 & ARMC 25 & 313 & $\mathrm{~S}$ \\
\hline heptaplatin/SK-2053R & 1999 & ARMC 35 & 348 & $\mathrm{~S}$ \\
\hline hexamethylmelamine & 1979 & FDA & & $\mathrm{S}$ \\
\hline hydroxyurea & 1968 & FDA & & $\mathrm{S}$ \\
\hline ifosfamide & 1976 & FDA & & $\mathrm{S}$ \\
\hline lenalidomide & 2005 & DNP 19 & 45 & $\mathrm{~S}$ \\
\hline levamisole & pre-1981 & Boyd & & $\mathrm{S}$ \\
\hline lobaplatin & 1998 & DNP 12 & 35 & $\mathrm{~S}$ \\
\hline lomustine (CCNU) & 1976 & FDA & & $\mathrm{S}$ \\
\hline lonidamine & 1987 & ARMC 23 & 337 & $\mathrm{~S}$ \\
\hline mechlorethanamine & 1958 & FDA & & $\mathrm{S}$ \\
\hline melphalan & 1961 & FDA & & $\mathrm{S}$ \\
\hline mitotane & 1970 & FDA & & $\mathrm{S}$ \\
\hline nedaplatin & 1995 & ARMC 31 & 347 & $\mathrm{~S}$ \\
\hline nilutamide & 1987 & ARMC 23 & 338 & $\mathrm{~S}$ \\
\hline nimustine hydrochloride & pre-1981 & Boyd & & $\mathrm{S}$ \\
\hline oxaliplatin & 1996 & ARMC 32 & 313 & $\mathrm{~S}$ \\
\hline pamidronate & 1987 & ARMC 23 & 326 & $\mathrm{~S}$ \\
\hline pipobroman & 1966 & FDA & & $\mathrm{S}$ \\
\hline porfimer sodium & 1993 & ARMC 29 & 343 & $\mathrm{~S}$ \\
\hline procarbazine & 1969 & FDA & & $\mathrm{S}$ \\
\hline ranimustine & 1987 & ARMC 23 & 341 & $\mathrm{~S}$ \\
\hline razoxane & pre-1977 & & & $\mathrm{S}$ \\
\hline semustine (MCCNU) & pre-1977 & & & $\mathrm{S}$ \\
\hline sobuzoxane & 1994 & ARMC 30 & 310 & $\mathrm{~S}$ \\
\hline sorafenib mesylate & 2005 & DNP 19 & 45 & $\mathrm{~S}$ \\
\hline thiotepa & 1959 & FDA & & $\mathrm{S}$ \\
\hline triethylenemelamine & pre-1981 & Boyd & & $\mathrm{S}$ \\
\hline zoledronic acid & 2000 & DNP 14 & 24 & $\mathrm{~S}$ \\
\hline anastrozole & 1995 & ARMC 31 & 338 & $\mathrm{~S} / \mathrm{NM}$ \\
\hline bicalutamide & 1995 & ARMC 31 & 338 & $\mathrm{~S} / \mathrm{NM}$ \\
\hline bortezomib & 2003 & ARMC 39 & 345 & $\mathrm{~S} / \mathrm{NM}$ \\
\hline camostat mesylate & 1985 & ARMC 21 & 325 & $\mathrm{~S} / \mathrm{NM}$ \\
\hline dasatinib $^{a}$ & 2006 & I 365055 & & $\mathrm{~S} / \mathrm{NM}$ \\
\hline erlotinib hydrochloride & 2004 & ARMC 40 & 454 & $\mathrm{~S} / \mathrm{NM}$ \\
\hline fadrozole $\mathrm{HCl}$ & 1995 & ARMC 31 & 342 & S/NM \\
\hline gefitinib & 2002 & ARMC 38 & 358 & $\mathrm{~S} / \mathrm{NM}$ \\
\hline imatinib mesilate & 2001 & DNP 15 & 38 & $\mathrm{~S} / \mathrm{NM}$ \\
\hline letrozole & 1996 & ARMC 32 & 311 & $\mathrm{~S} / \mathrm{NM}$ \\
\hline nafoxidine & pre-1977 & & & $\mathrm{S} / \mathrm{NM}$ \\
\hline sunitinib maleate & 2006 & I 309144 & & $\mathrm{~S} / \mathrm{NM}$ \\
\hline tamoxifen & 1973 & FDA & & S/NM \\
\hline toremifene & 1989 & ARMC 25319 & & $\mathrm{~S} / \mathrm{NM}$ \\
\hline aminoglutethimide & 1981 & FDA & & $\mathrm{S}^{*}$ \\
\hline azacytidine & pre-1977 & & & $\mathrm{S}^{*}$ \\
\hline capecitabine & 1998 & ARMC 34 & 319 & $\mathrm{~S}^{*}$ \\
\hline carmofur & 1981 & FDA & & $\mathrm{S}^{*}$ \\
\hline clofarabine & 2005 & DNP 19 & 44 & $\mathrm{~S}^{*}$ \\
\hline
\end{tabular}


Table 9. Continued

\begin{tabular}{|c|c|c|c|c|}
\hline generic name & year introduced & reference & page & source \\
\hline cytosine arabinoside & 1969 & FDA & & $\mathrm{S}^{*}$ \\
\hline decitabine & 2006 & I 125366 & & $\mathrm{~S}^{*}$ \\
\hline doxifluridine & 1987 & ARMC 23 & 332 & $\mathrm{~S}^{*}$ \\
\hline enocitabine & 1983 & ARMC 19 & 318 & $\mathrm{~S}^{*}$ \\
\hline floxuridine & 1971 & FDA & & $\mathrm{S}^{*}$ \\
\hline fludarabine phosphate & 1991 & ARMC 27 & 327 & $\mathrm{~S}^{*}$ \\
\hline fluorouracil & 1962 & FDA & & $\mathrm{S}^{*}$ \\
\hline ftorafur & 1972 & FDA & & $\mathrm{S}^{*}$ \\
\hline gemcitabine $\mathrm{HCl}$ & 1995 & ARMC 31 & 344 & $\mathrm{~S}^{*}$ \\
\hline mercaptopurine & 1953 & FDA & & $\mathrm{S}^{*}$ \\
\hline methotrexate & 1954 & FDA & & $\mathrm{S}^{*}$ \\
\hline mitoxantrone $\mathrm{HCI}$ & 1984 & ARMC 20 & 321 & $\mathrm{~S}^{*}$ \\
\hline nelarabine & 2005 & DNP 19 & 45 & $\mathrm{~S}^{*}$ \\
\hline thioguanine & 1966 & FDA & & $\mathrm{S}^{*}$ \\
\hline uracil mustard & 1966 & FDA & & $\mathrm{S}^{*}$ \\
\hline abarelix & 2004 & ARMC 40 & 446 & $\mathrm{~S} * / \mathrm{NM}$ \\
\hline bexarotene & 2000 & DNP 14 & 23 & $\mathrm{~S} * / \mathrm{NM}$ \\
\hline pemetrexed & 2004 & ARMC 40 & 463 & $\mathrm{~S} * / \mathrm{NM}$ \\
\hline raltitrexed & 1996 & ARMC 32 & 315 & $\mathrm{~S} * / \mathrm{NM}$ \\
\hline tamibarotene & 2005 & DNP 19 & 45 & $\mathrm{~S} * / \mathrm{NM}$ \\
\hline temozolomide & 1999 & ARMC 35 & 350 & $\mathrm{~S} * / \mathrm{NM}$ \\
\hline bcg live & 1990 & DNP 04 & 104 & $\mathrm{~V}$ \\
\hline melanoma theraccine & 2001 & DNP 15 & 38 & $\mathrm{~V}$ \\
\hline
\end{tabular}

${ }^{a}$ One extra drug added, approved June 28, 2006, launched July 3, 2006.

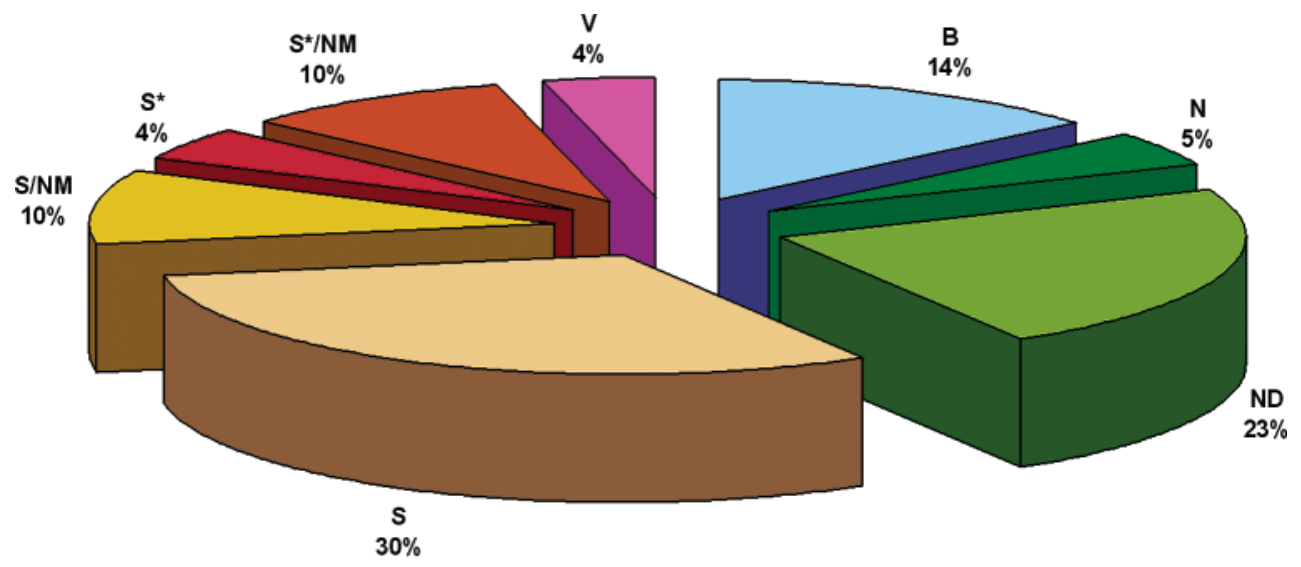

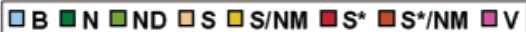

Figure 1. All new chemical entities, $01 / 1981-06 / 2006$, by source $(N=1184)$.

or hematologic stimulants. Etoposide phosphate is not included in this count, as it is a prodrug of etoposide, though it was included in our last review as an approved NCE. We have however included paclitaxel nanoparticles, as this is not just a salt form but is a novel form of the agent ensuring much better water solubility.

In our earlier papers, the number of nonsynthetic antitumor agents was $62 \%$ for other than biologicals/vaccines, without an "NM" subcategory. The corresponding figure obtained by removing the NM subcategory in this analysis is $64 \%$. Thus, the proportion has remained similar in spite of some reassignments of sources and the expansion of combinatorial chemistry techniques. As mentioned earlier, the first and only de novo combinatorial drug that we have been able to identify was approved by the FDA in 2005 under the generic name of sorafenib mesylate (1) for the treatment of advanced renal cancer.

A major general class of drugs that was not commented on in any detail in our earlier papers is the class that is directed toward the treatment of diabetes, both types I and II (Table 10; $n=32$ ). These drugs include a significant number of biologics based upon varying modifications of insulin produced in general by biotechnological means $(\mathrm{B}, 18 ; 56.3 \%) .{ }^{50}$ In addition to these well-known agents, the class also includes a very interesting compound (approved by the FDA in 2005) that is assigned to the ND class (extenatide or Byetta). This is the first in a new class of therapeutic agents known as incretin mimetics. The drug exhibits glucoselowering activity similar to the naturally occurring incretin hormone glucagon-like peptide-1 (GLP-1), but is a 39-residue peptide based upon one of the peptide venoms of the Gila monster, Heloderma suspectum. ${ }^{77}$

\section{Discussion}

As alluded to in our previous review, the decline or leveling of the output of the R\&D programs of the pharmaceutical companies has continued, with the number of drugs of all types dropping in 2003 to 35 launches, including 13 in the B/V categories, and reaching a nadir in 2004, when only 25 were launches and 6 of these fell into the $\mathrm{B} / \mathrm{V}$ categories. There was a significant upswing in 2005 with 54 launches, but 20 of these were in the B/V categories, leaving 34 small molecules. In the first 6 months of 2006 , of the 22 launches, 9 were B/V.

Although combinatorial chemistry continues to play a major role in the drug development process, as mentioned earlier, it is noteworthy that the trend toward the synthesis of complex natural product-like libraries has continued. As was eloquently stated by Danishefsky in 2002, "a small collection of smart compounds may be more valuable than a much larger hodgepodge collection mindlessly assembled". ${ }^{78}$ Recently he and a coauthor restated this theme: ${ }^{79}$ 


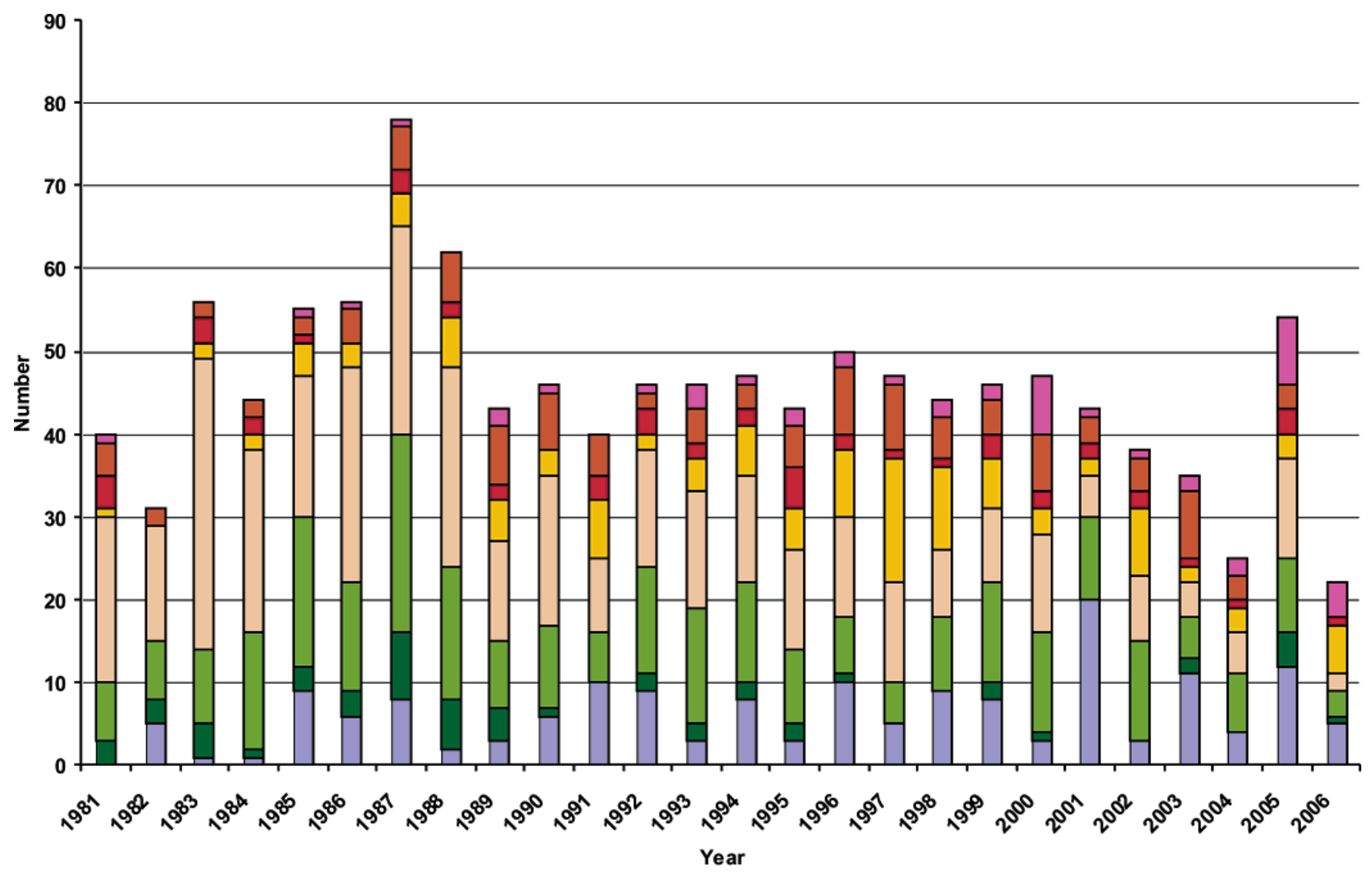

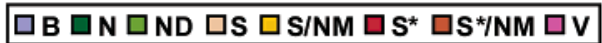

Figure 2. All new chemical entities organized by source/year $(N=1184)$.

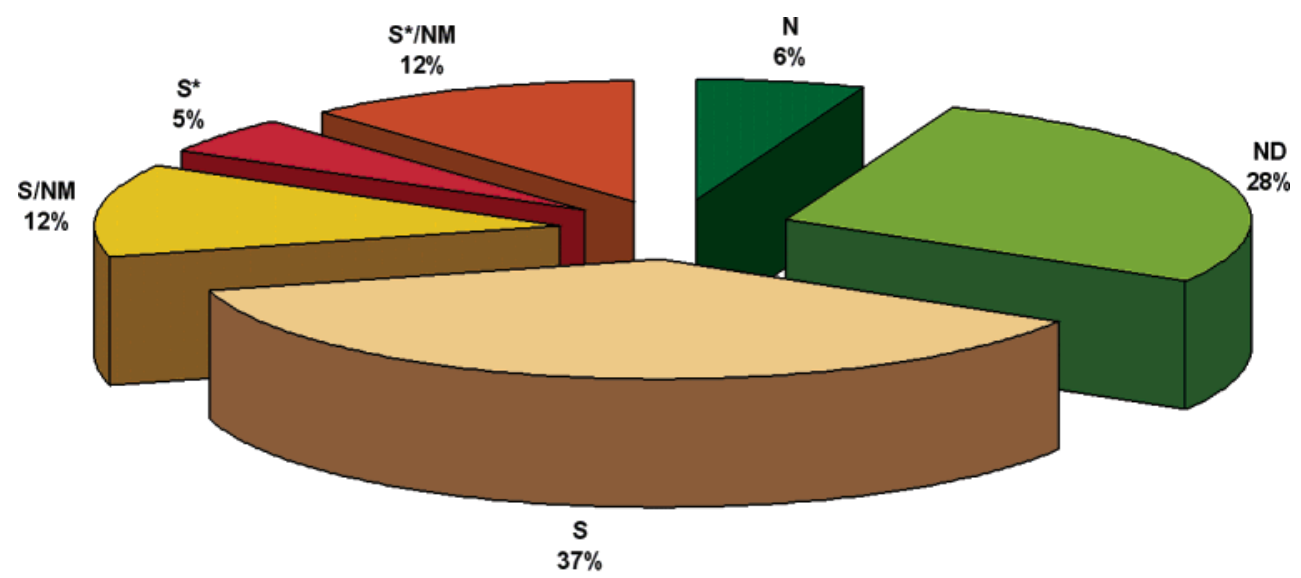

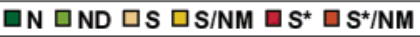

Figure 3. All small molecule new chemical entities, $01 / 1981-06 / 2006$, by Source $(N=974)$.

In summary, we have presented several happy experiences in the course of our program directed toward bringing to bear nature's treasures of small molecule natural products on the momentous challenge of human neurodegenerative diseases. While biological results are now being accumulated for systematic disclosure, it is already clear that there is considerable potential in compounds obtained through plowing in the landscape of natural products. Particularly impressive are those compounds that are obtained through diverted total synthesis, i.e., through methodology, which was redirected from the original (and realized) goal of total synthesis, to encompass otherwise unavailable congeners. We are confident that the program will lead, minimally, to compounds that are deserving of serious preclinical follow-up. At the broader level, we note that this program will confirm once again (if further confirmation is, indeed, necessary) the extraordinary advantages of small molecule natural products as sources of agents, which interject themselves in a helpful way in various physiological processes.

We close with the hope and expectation that enterprising and hearty organic chemists will not pass up the unique head start that natural products provide in the quest for new agents and new directions in medicinal discovery. We would chance to predict that even as the currently fashionable "telephone directory" mode of research is subjected to much overdue scrutiny and performance-based assessment, organic chemists in concert with biologists and even clinicians will be enjoying as well as exploiting the rich troves provided by nature's small molecules. 


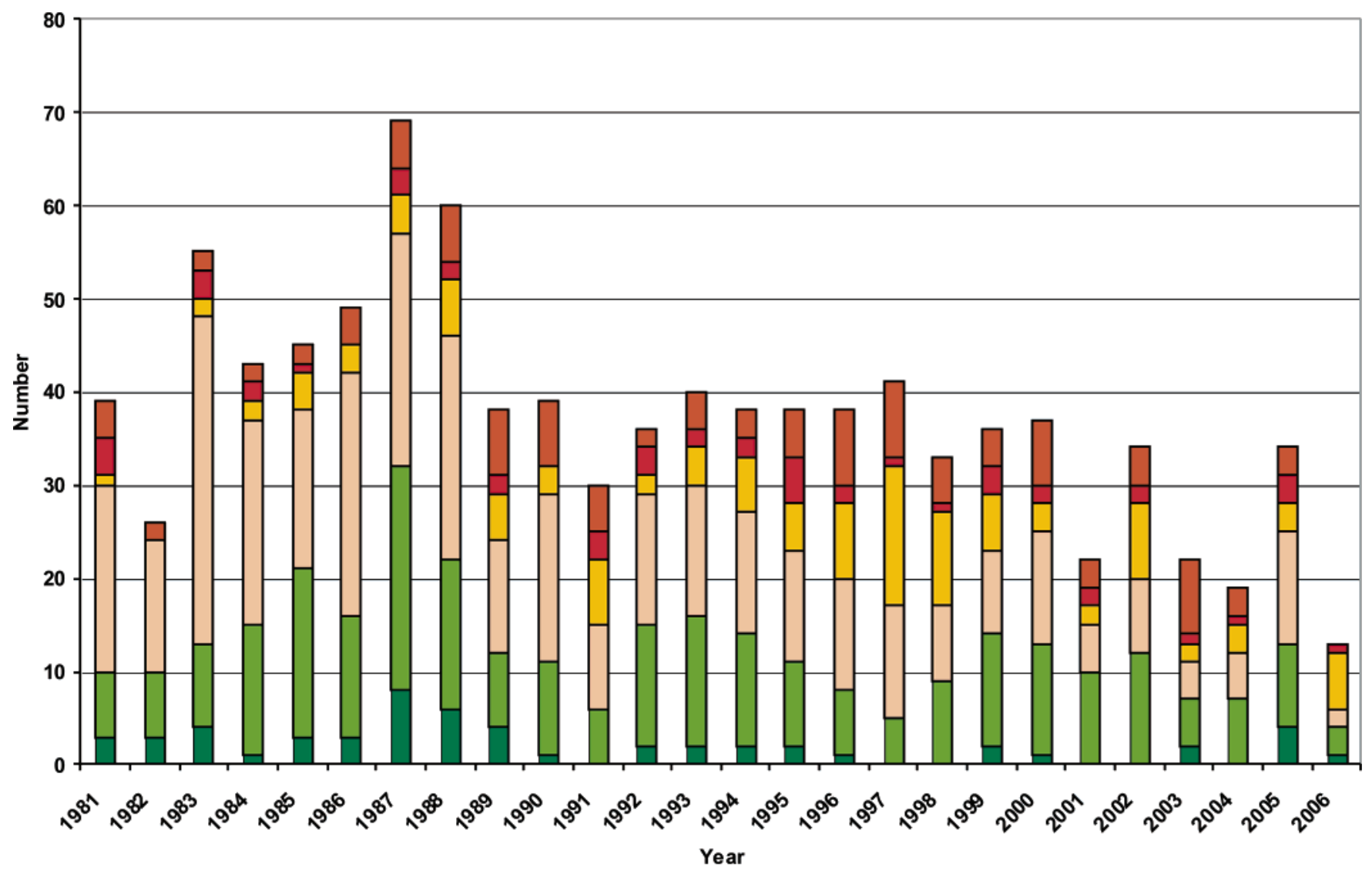

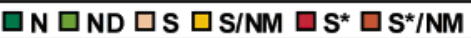

Figure 4. Small molecule new chemical entities organized by source/year $(N=974)$.
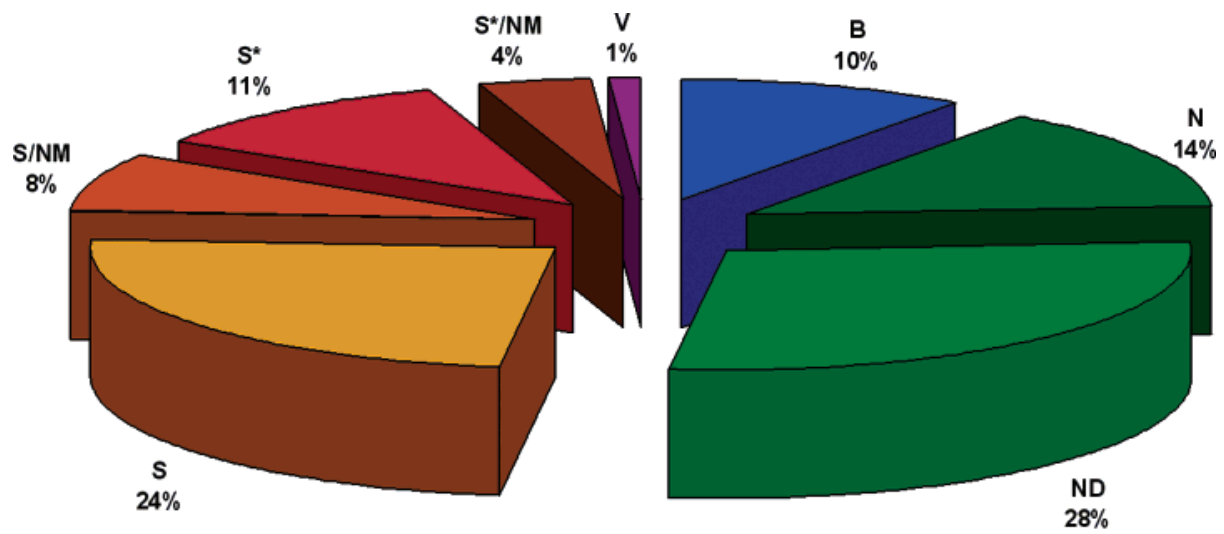

$\square B \square N \square N D \square S \square S / N M \square S^{\star} \square S^{\star} / N M \square V$

Figure 5. All available anticancer drugs, $1940 s-06 / 2006$, by source $(N=175)$.

A rapid analysis of the entities approved from 2003 to 2006 (the full data set is available as an Excel spreadsheet in the Supporting Information) indicated that there were significant numbers of antitumor, antibacterial, and antifungal agents approved as mentioned above. This time frame also saw two very important approvals, both of which were natural products. The first was the approval by the FDA, after a long series of trials and discussions, of the cone snail toxin known as Prialt, which is the first "direct from the sea" entity to become a licensed pharmaceutical. ${ }^{80,81}$ Although one can argue (as we have on other occasions) that the discovery of the arabinose nucleosides by Bergmann in the 1950s was the driving force behind Ara-A, Ara-C, AZT, etc., this is the first direct transition from marine invertebrate to man. Also in the middle of 2006, the botanical preparation Hemoxin ${ }^{82,83}$ was approved in Nigeria following demonstration of efficacy in clinical trials as a treatment for sickle cell anemia. This is a mix of plants that came from native healer information and thus can be classified as a "true ethnobotanical preparation".

In this paper, as we stated in $2003,{ }^{2}$ we have again demonstrated that natural products play a dominant role in the discovery of leads for the development of drugs for the treatment of human diseases. Some have argued (though not in press, only in personal conversations at various fora) that the introduction of categories such as $\mathrm{S} / \mathrm{NM}$ and $\mathrm{S} * / \mathrm{NM}$ is an overstatement of the role played by natural products in the drug discovery process. On the contrary, we would argue that these further serve to illustrate the inspiration provided by Nature to receptive organic chemists in devising ingenious syntheses of structural mimics to compete with Mother Nature's 


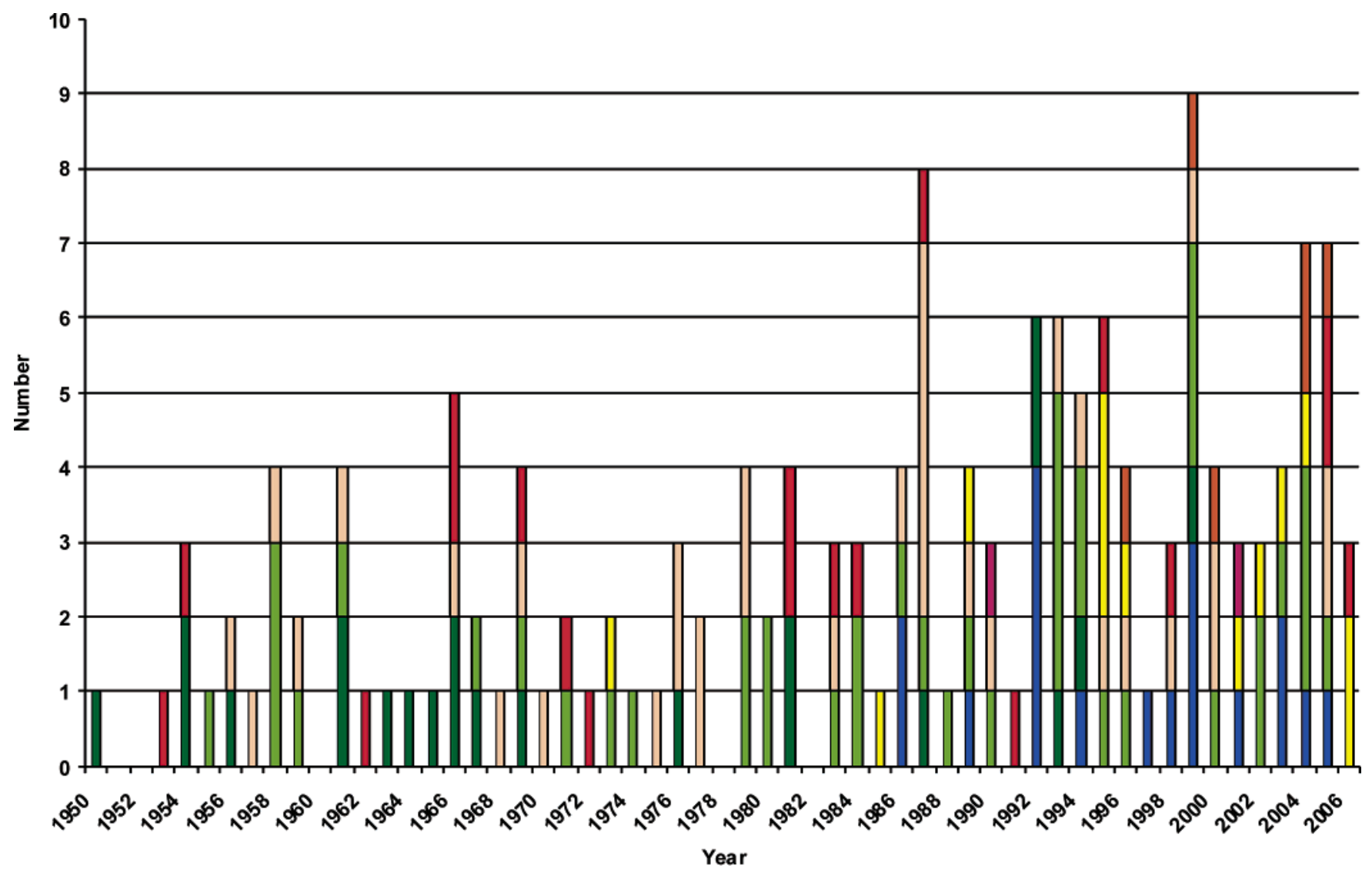

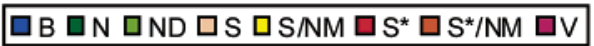

Figure 6. Approved anticancer agents, organized by source/year (known dates for 157).

Table 10. Antidiabetic Drugs from 01/1981 to 06/2006 Organized Alphabetically by Generic Name within Source

\begin{tabular}{|c|c|c|c|c|c|}
\hline generic name & trade name & year introduced & reference & page & source \\
\hline biphasic porcine insulin & Pork Mixtard 30 & 1982 & I 303034 & & B \\
\hline hu neutral insulin & Insuman & 1992 & I 255451 & & $\mathrm{~B}$ \\
\hline human insulin $\mathrm{Zn}$ suspension & Humulin L & 1985 & I 302828 & & $\mathrm{~B}$ \\
\hline human insulin zinc suspension & Humulin Zn & 1985 & I 091584 & & $\mathrm{~B}$ \\
\hline human neutral insulin & Novolin R & 1991 & I 182551 & & $\mathrm{~B}$ \\
\hline insulin aspart & NovoRapid & 1999 & DNP 13 & 41 & B \\
\hline insulin aspart/IA protamine & NovoMix 30 & 2001 & DNP 15 & 34 & $\mathrm{~B}$ \\
\hline insulin determir & Levemir & 2004 & DNP 18 & 27 & $\mathrm{~B}$ \\
\hline insulin glargine & Lantus & 2000 & DNP 14 & 19 & $\mathrm{~B}$ \\
\hline insulin glulisine & Apidra & 2005 & DNP 19 & 39 & $\mathrm{~B}$ \\
\hline insulin lispro & Humalog & 1996 & ARMC 32 & 310 & B \\
\hline isophane insulin & Humulin $\mathrm{N}$ & 1982 & I 091583 & & $\mathrm{~B}$ \\
\hline mecasermin & Somazon & 1994 & DNP 08 & 28 & B \\
\hline oral insulin & Oral-lyn & 2005 & DNP 19 & 39 & $\mathrm{~B}$ \\
\hline porcine isophane insulin & Pork Insulatard & 1982 & I 302757 & & $\mathrm{~B}$ \\
\hline porcine neutral insulin & Pork Actrapid & 1998 & I 302749 & & B \\
\hline pulmonary insulin & Exubera & 2005 & I 229896 & & $\mathrm{~B}$ \\
\hline soluble insulin & Velosulin BR & 1986 & I 091581 & & B \\
\hline voglibose & Basen & 1994 & ARMC 30 & 313 & $\mathrm{~N}$ \\
\hline acarbose & Glucobay & 1990 & DNP 03 & 23 & ND \\
\hline extenatide & Byetta & 2005 & DNP 19 & 40 & ND \\
\hline miglitol & Diastabol & 1998 & ARMC 34 & 325 & ND \\
\hline triproamylin acetate & Normylin & 2005 & DNP 19 & 40 & ND \\
\hline glimepiride & Amaryl & 1995 & ARMC 31 & 344 & $\mathrm{~S}$ \\
\hline mitiglinide calcium hydrate & Glufast & 2004 & ARMC 40 & 460 & S \\
\hline pioglitazone $\mathrm{NCl}$ & Actos & 1999 & ARMC 35 & 346 & $\mathrm{~S}$ \\
\hline repaglinide & Prandin & 1998 & ARMC 34 & 329 & $\mathrm{~S}$ \\
\hline epalrestat & Kinedak & 1992 & ARMC 28 & 330 & S/NM \\
\hline rosiglitazone maleate & Avandia & 1999 & ARMC 35 & 348 & S/NM \\
\hline tolrestat & Alredase & 1989 & ARMC 25 & 319 & S/NM \\
\hline troglitazone & Rezulin & 1997 & ARMC 33 & 344 & S/NM \\
\hline nateglinide & Starsis & 1999 & ARMC 35 & 344 & $\mathrm{~S}^{*}$ \\
\hline
\end{tabular}

longstanding substrates. Even discounting these categories, the continuing and overwhelming contribution of natural products to the expansion of the chemotherapeutic armamentarium is clearly evident, and as we stated in our earlier papers, much of Nature's "treasure trove of small molecules" remains to be explored, particularly from the marine and microbial environments. 


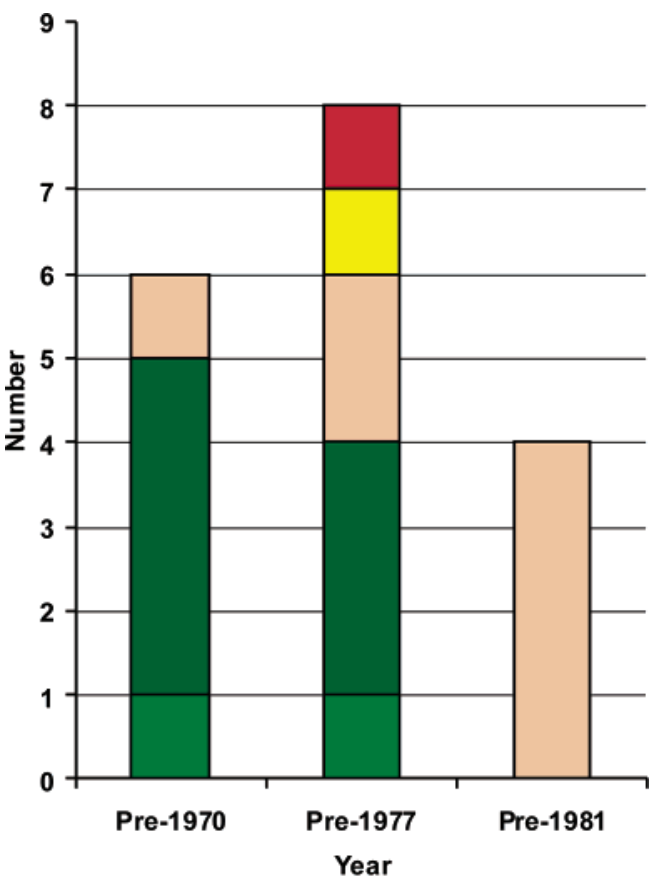

$\square \mathrm{N} \square \mathrm{ND} \square \mathrm{S} \square \mathrm{S} / \mathrm{NM} \square \mathrm{S}^{*} \square \mathrm{S}^{*} / \mathrm{NM}$

Figure 7. Approved anticancer agents, organized by source/year (unknown dates for 18).

From the perspective of microbes and their role(s) as sources of novel bioactive entities, the recent work that has been reported by a variety of investigators as to the potential of these organisms needs to be widely disseminated. Over the last few years, it has become obvious from analyses of the published (and, to some extent, unpublished) genomic sequences of a variety of microbes that there are at least a dozen potential biosynthetic clusters in each organism surveyed and, in certain well-publicized cases, over 30 such groupings. ${ }^{84-92}$ In the marine environment the interplay of these two sources, as exemplified by the recent review by Newman and Hill, ${ }^{93}$ leaves no doubt that a host of novel, bioactive chemotypes await discovery from both terrestrial and marine sources.

In this respect it should be noted that in the last year or so there has been a very significant series of findings where the well-known antitumor agents camptothecin ${ }^{94}$ and podophyllotoxin ${ }^{95}$ and vincristine $^{96}$ have now been produced by fermentation of endophytic fungi, isolated from the producing plants. The usual argument that these are artifacts because of the inability to produce large quantities by regular fermentation processes has been shown to be specious by the work by Bok et al. ${ }^{84}$ with Aspergillus nidulans. This work demonstrated that one has to be able to find the "genetic on switch" to be able to obtain expression of such clusters outside of the host. In addition to these papers the reader's attention is also drawn to the recent excellent review article by Gunatilaka ${ }^{97}$ on this subject, which gives an excellent overview of the numbers of materials so far discovered from these sources. As a result, investigators need to consider all possible routes to novel agents.

To us, a multidisciplinary approach to drug discovery, involving the generation of truly novel molecular diversity from natural product sources, combined with total and combinatorial synthetic methodologies, and including the manipulation of biosynthetic pathways (so-called combinatorial biosynthesis), provides the best solution to the current productivity crisis facing the scientific community engaged in drug discovery and development.

Once more, as we stated in our 2003 review, ${ }^{2}$ we strongly advocate expanding, not decreasing, the exploration of Nature as a source of novel active agents that may serve as the leads and scaffolds for elaboration into desperately needed efficacious drugs for a multitude of disease indications.
Supporting Information Available: An Excel XP spreadsheet is available free of charge via the Internet at http://pubs.acs.org.

\section{References and Notes}

(1) Cragg, G. M.; Newman, D. J.; Snader, K. M. J. Nat. Prod. 1997, $60,52-60$

(2) Newman, D. J.; Cragg, G. M.; Snader, K. M. J. Nat. Prod. 2003 , 66, $1022-1037$.

(3) Pelish, H. E.; Westwood, N. J.; Feng, Y.; Kirchausen, T.; Shair, M. D. J. Am. Chem. Soc. 2001, 123, 6740-6741.

(4) Spring, D. R. Org. Biomol. Chem. 2003, 1, 3867-3870.

(5) Burke, M. D.; Schreiber, S. L. Angew. Chem., Int. Ed. 2004, 43, $46-58$

(6) Zhonghong, G.; Reddy, P. T.; Quevillion, S.; Couve-Bonnaire, S.; Ayra, P. Angew. Chem., Int. Ed. 2005, 44, 1366-1368.

(7) Reayi, A.; Arya, P. Curr. Opin. Chem. Biol. 2005, 9, 240-247.

(8) Lipinski, C. A. Drug Discovery Today: Technol. 2004, 1, 337341.

(9) Macarron, R. Drug Discovery Today 2006, 11, 277-279.

(10) Keller, T. H.; Pichota, A.; Yin, Z. Curr. Opin. Chem. Biol. 2006, 10, 357-361.

(11) Allen, R. C. In Annual Reports in Medicinal Chemistry; Bailey, D. M., Ed.; Academic Press: Orlando, 1984; Vol. 19, pp 313-326.

(12) Allen, R. C. In Annual Reports in Medicinal Chemistry; Bailey, D. M., Ed.; Academic Press: Orlando, 1985; Vol. 20, pp 315-325.

(13) Allen, R. C. In Annual Reports in Medicinal Chemistry; Bailey, D. M., Ed.; Academic Press: Orlando, 1986; Vol. 21, pp 323-335.

(14) Allen, R. C. In Annual Reports in Medicinal Chemistry; Bailey, D. M., Ed.; Academic Press: Orlando, 1987; Vol. 22, pp 315-330.

(15) Ong, H. H.; Allen, R. C. In Annual Reports in Medicinal Chemistry; Allen, R. C., Ed.; Academic Press: San Diego, 1988; Vol. 23, pp 325- 348 .

(16) Ong, H. H.; Allen, R. C. In Annual Reports in Medicinal Chemistry; Allen, R. C., Ed.; Academic Press: San Diego, 1989; Vol. 24, pp $295-315$.

(17) Ong, H. H.; Allen, R. C. In Annual Reports in Medicinal Chemistry; Bristol, J. A., Ed.; Academic Press: San Diego, 1990; Vol. 25, pp 309-322.

(18) Strupczewski, J. D.; Ellis, D. B.; Allen, R. C. In Annual Reports in Medicinal Chemistry; Bristol, J. A., Ed.; Academic Press: San Diego, 1991; Vol. 26, pp 297-313.

(19) Strupczewski, J. D.; Ellis, D. B. In Annual Reports in Medicinal Chemistry; Bristol, J. A., Ed.; Academic Press: San Diego, 1992; Vol. 27, pp 321-337.

(20) Strupczewski, J. D.; Ellis, D. B. In Annual Reports in Medicinal Chemistry; Bristol, J. A., Ed.; Academic Press: San Diego, 1993; Vol. 28, pp 325-341.

(21) Cheng, X.-M. In Annual Reports in Medicinal Chemistry; Bristol, J. A., Ed.; Academic Press: San Diego, 1994; Vol. 29, pp 331-354.

(22) Cheng, X.-M. In Annual Reports in Medicinal Chemistry; Bristol, J. A., Ed.; Academic Press: San Diego, 1995; Vol. 30, pp 295-317.

(23) Cheng, X.-M. In Annual Reports in Medicinal Chemistry; Bristol, J. A., Ed.; Academic Press: San Diego, 1996; Vol. 31, pp 337-355.

(24) Galatsis, P. In Annual Reports in Medicinal Chemistry; Bristol, J. A., Ed.; Academic Press: San Diego, 1997; Vol. 32, pp 305-326.

(25) Galatsis, P. In Annual Reports in Medicinal Chemistry; Bristol, J. A., Ed.; Academic Press: San Diego, 1998; Vol. 33, pp 327-353.

(26) Gaudilliere, B. In Annual Reports in Medicinal Chemistry; Doherty, A. M., Ed.; Academic Press: San Diego, 1999; Vol. 34, pp 317338.

(27) Gaudilliere, B.; Berna, P. In Annual Reports in Medicinal Chemistry; Doherty, A. M., Ed.; Academic Press: San Diego, 2000; Vol. 35, pp 331-355.

(28) Gaudilliere, B.; Bernardelli, P.; Berna, P. In Annual Reports in Medicinal Chemistry; Doherty, A. M., Ed.; Academic Press: San Diego, 2001; Vol. 36, pp 293-318.

(29) Bernardelli, P.; Gaudilliere, B.; Vergne, F. In Annual Reports in Medicinal Chemistry; Doherty, A. M., Ed.; Academic Press: Amsterdam, 2002; Vol. 37, pp 257-277.

(30) Boyer-Joubert, C.; Lorthiois, E.; Moreau, F. In Annual Reports in Medicinal Chemistry; Doherty, A. M., Ed.; Academic Press: Amsterdam, 2003; Vol. 38, pp 347-374.

(31) Hegde, S.; Carter, J. In Annual Reports in Medicinal Chemistry; Doherty, A. M., Ed.; Academic Press: Amsterdam, 2004; Vol. 39, pp 337-368.

(32) Hegde, S.; Schmidt, M. In Annual Reports in Medicinal Chemistry; Doherty, A. M., Ed.; Academic Press: Amsterdam, 2005; Vol. 40, pp 443-473.

(33) Prous, J. R. Drug News Perspect. 1990, 3, 19-29.

(34) Prous, J. R. Drug News Perspect. 1991, 4, 96-109.

(35) Prous, J. R. Drug News Perspect. 1992, 5, 93-101.

(36) Prous, J. R. Drug News Perspect. 1993, 6, 95-106.

(37) Prous, J. R. Drug News Perspect. 1994, 7, 26-36. 
(38) Prous, J. R. Drug News Perspect. 1995, 8, 24-37.

(39) Prous, J. R. Drug News Perspect. 1996, 9, 19-32.

(40) Graul, A. I. Drug News Perspect. 1997, 10, 5-18.

(41) Graul, A. I. Drug News Perspect. 1998, 11, 15-32.

(42) Graul, A. I. Drug News Perspect. 1999, 12, 27-43.

(43) Graul, A. I. Drug News Perspect. 2000, 13, 37-53.

(44) Graul, A. I. Drug News Perspect. 2001, 14, 12-31.

(45) Graul, A. I. Drug News Perspect. 2002, 15, 29-43.

(46) Graul, A. I. Drug News Perspect. 2003, 16, 22-39.

(47) Graul, A. I. Drug News Perspect. 2004, 17, 43-57.

(48) Graul, A. I. Prous, J. R., Drug News Perspect. 2005, 18, 21-36.

(49) Graul, A. I. Prous, J. R., Drug News Perspect. 2006, 19, 33-53.

(50) Newman, D. J.; Cragg, G. M.; O’Keefe, B. R. In Modern Biopharmaceuticals, Design, Development and Optimization; Knablein, J., Ed.; Wiley-VCH: Weinheim, 2005; Vol. 2, pp 451-496.

(51) Boyd, M. R. In Current Therapy in Oncology; Neiderhuber, J., Ed.; Decker: Philadelphia, 1993; pp 11-22.

(52) Sweetman, S. C. Martindale, The Complete Drug Reference, 33 ed.; The Pharmaceutical Press: London, 2002.

(53) Hruby, V. J. Nat. Rev., Drug Discovery 2002, 1, 847-858.

(54) Wan, Y.; Wallinder, C.; Plouffe, B.; Beaudry, H.; Mahalingam, A K.; Wu, X.; Johansson, B.; Holm, M.; Botros, M.; Karlen, A.; Petterson, A.; Nyberg, F.; Fandricks, L.; Gallo-Payet, N.; Hallberg, A.; Alterman, M. J. Med. Chem. 2004, 47, 5995-6908.

(55) Georgsson, J.; Rosenstrom, U.; Wallinder, C.; Beaudry, H.; Plouffe, B.; Lindeberg, G.; Botros, M.; Nyberg, F.; Karlen, A.; Gallo-Payet, N.; Hallberg, A. Bioorg. Med. Chem. 2006, 14, 5963-5972.

(56) Steckelings, U. M.; Kaschina, E.; Unger, T. Peptides 2005, 26, 1401 1409.

(57) Newman, D. J.; Cragg, G. M.; Snader, K. M. Nat. Prod. Rep. 2000 , $17,215-234$

(58) Breinbauer, R.; Manger, M.; Scheck, M.; Waldmann, H. Curr. Med. Chem. 2002, 9, 2129-2145.

(59) Breinbauer, R.; Vetter, I. R.; Waldmann, H. Angew. Chem., Int. Ed. 2002, 41, 2878-2890.

(60) Kingston, D. G. I.; Newman, D. J. Curr. Opin. Drug Discovery Dev. 2002, 5, 304-316.

(61) Newman, D. J.; Cragg, G. M.; Holbeck, S.; Sausville, E. A. Curr Cancer Drug Targets 2002, 2, 279-308.

(62) Nielsen, J. Curr. Opin. Chem. Biol. 2002, 6, 297-305.

(63) Perez, J. J.; Corcho, F.; Llorens, O. Curr. Med. Chem. 2002, 9, 22092229.

(64) van Huijsduijnen, R. H.; Bombrun, A.; Swinnen, D. Drug Discovery Today 2002, 7, 1013-1019.

(65) Barun, O.; Sommer, S.; Waldmann, H. Angew. Chem., Int. Ed. 2004, 43, 3195-3199.

(66) Balamurugan, R.; Dekker, F. J.; Waldmann, H. Mol. BioSyst. 2005, $1,36-45$.

(67) Ganesan, A. Curr. Opin. Biotech. 2004, 15, 584-590.

(68) Shang, S.; Tan, D. S. Curr. Opin. Chem. Biol. 2005, 9, 248-258.

(69) Costantino, L.; Barlocco, D. Curr. Med. Chem. 2006, 13, 65-85.

(70) Violette, A.; Fournel, S.; Frisch, B.; Briand, J.-P.; Monteil, H.; Guichard, G. Chem. Biol. 2006, 13, 531-538.
(71) Baltz, R. H.; Miao, V.; Wrigley, S. K. Nat. Prod. Rep. 2005, 22, 717-741

(72) Melby, T.; Sista, P.; DeMasi, R.; Kirkland, T.; Roberts, N.; Salgo, M.; Heilek-Snyder, G.; Cammack, N.; Matthews, T. J.; Greenberg, M. L. AIDS Res. Hum. Retroviruses 2006, 22, 375-385.

(73) Yang, S. S.; Cragg, G. M.; Newman, D. J.; Bader, J. P. J. Nat. Prod. 2001, 64, 265-277.

(74) Efferth, T. Drug Res. Updates 2005, 8, 85-97.

(75) Carter, S. K.; Bakowski, M. T.; Hellmann, K. Chemotherapy of Cancer; Wiley: New York, 1977; p 350.

(76) Cole, W. H. Chemotherapy of Cancer; Lea and Febiger: Philadelphia, 1970; p 349 .

(77) Iltz, J. L.; Baker, D. E.; Setter, S. M.; Campbell, R. K. Clin. Ther 2006, 28, 652-665.

(78) Borman, S. Chem. Eng. News 2002, Jan 14, 23-24.

(79) Wilson, R. M.; Danishefsky, S. J. Acc. Chem. Res. 2006, 39, 539 -549 .

(80) Klotz, U. Int. J. Clin. Pharmacol. Ther. 2006, 44, 478-483.

(81) Wermeling, D. P.; Berger, J. R. Pharmacotherapy 2006, 26, 395402.

(82) Iyamu, E. W.; Turner, E. A.; Asakura, T. Br. J. Haematol. 2003, $122,1001-1008$

(83) Cordeiro, N. J.; Oniyangi, O. Cochrane Database Syst. Rev. 2004 CD004448.

(84) Bok, J. W.; Hoffmeister, D.; Maggio-Hall, L. A.; Murillo, R.; Glasner, J. D.; Keller, N. P. Chem. Biol. 2006, 13, 31-37.

(85) Challis, G. L.; Ravel, J. FEMS Microbiol. Lett. 2000, 187, 111114.

(86) Lautru, S.; Deeth, R. J.; Bailey, L. M.; Challis, G. L. Nat. Chem. Biol. 2005, 1, 265-269.

(87) McAlpine, J. B.; Bachmann, B. O.; Piraee, M.; Tremblay, S.; Alarco, A.-M.; Zazopoulos, E.; Farnet, C. M. J. Nat. Prod. 2005, 68, 493496.

(88) Piel, J. Nat. Prod. Rep. 2004, 21, 519-538.

(89) Piel, J. BioSpektrum 2005, 11, 172-173.

(90) Piel, J. Curr. Med. Chem. 2006, 13, 39-50.

(91) Piel, J.; Butzke, D.; Fusetani, N.; Hui, D.; Platzer, M.; Wen, G.; Matsunaga, S. J. Nat. Prod. 2005, 68, 472-479.

(92) Piel, J.; Hui, D.; Wen, G.; Butzke, D.; Platzer, M.; Fusetani, N.; Matsunaga, S. Proc. Natl. Acad. Sci. U.S.A. 2004, 101, 16222-16227.

(93) Newman, D. J.; Hill, R. T. J. Ind. Microbiol. Biotechnol. 2006, 23, $539-544$

(94) Puri, S. C.; Verma, V.; Amna, T.; Qazi, G. N.; Spiteller, M. J. Nat. Prod. 2005, 68, 1717-1719.

(95) Eyberger, A. L.; Dondapati, R.; Porter, J. R. J. Nat. Prod. 2006, 69, $1121-1124$.

(96) Yang, X.; Zhang, L.; Guo, B.; Guo, S. Zhong Cao Yao 2004, 35, $79-81$.

(97) Gunatilaka, A. A. L. J. Nat. Prod. 2006, 69, 509-526.

NP068054V 